\title{
On involute-evolute curve couple in the hyperbolic and de Sitter spaces
}

\author{
H. S. Abdel-Aziz ${ }^{1}$, M. Khalifa Saad ${ }^{1,2^{*}}$ (1) and A. A. Abdel-Salam ${ }^{1}$
}

\author{
${ }^{*}$ Correspondence: \\ m_khalifag@yahoo.com; \\ mohamed_khalifa77@science. \\ sohag.edu.eg \\ 'Department of Mathematics, \\ Faculty of Science, Sohag University, \\ Naser Street, Sohag, 82524, Egypt \\ 2 Department of Mathematics, \\ Faculty of Science, Islamic \\ University of Madinah, 170 \\ Madinah, Kingdom of Saudi Arabia
}

\begin{abstract}
This paper aims at showing that Frenet apparatus of an evolute curve can be formed in terms of Frenet apparatus of its involute curve in the hyperbolic (de Sitter) space. Also, we establish relationships among Frenet frame of the considered curve couple. Finally, we defray some examples to confirm our main results.
\end{abstract}

Keywords: Frenet frames, Involute-evolute curves, Hyperbolic space

MSC: $53 \mathrm{~A} 25,53 \mathrm{C} 50$

\section{Introduction}

The idea of a string involute is due to Christian Huygens (1658), who is also known for his work in optics. He discovered involutes while trying to develop a more accurate clock [1]. The involute of a given curve is a well-known concept in Euclidean 3-space $R^{3}$. It is well-known that if a curve is differentiable at each point of an open interval, a set of mutually orthogonal unit vectors can be constructed and called Frenet frame or moving frame vectors. The rates of these frame vectors along the curve define curvatures of the curves. The set, whose elements are frame vector and curvatures of a curve, is called Frenet apparatus of the curve.

It is safe to report that the many important results in the theory of the curves in $R^{3}$ were initiated by G. Monge, and G. Darboux pioneered the moving frame idea (for more details see [2]). Thereafter, Frenet defined his moving frame and his special equations which play important role in mechanics and kinematics as well as in differential geometry. At the beginning of the twentieth century, A. Einstein's theory opened a door to the use of new geometries. One of them, Minkowski space-time, which is simultaneously the geometry of special relativity and the geometry induced on each fixed tangent space of an arbitrary Lorentzian manifold, was introduced, and some of the classical differential geometry topics have been treated by the researchers. In the recent years, the theory of degenerate submanifolds has been treated by researchers and some classical differential geometry topics have been extended to Lorentz manifolds. For instance, in [3-5], the authors extended and studied spacelike involute-evolute curves in Euclidean 4-space and Minkowski space-time.

An evolute and its involute are defined in mutual pairs. The evolute and the involute of the curve pair are well known by the mathematicians especially the differential geometry scientists. The evolute of any curve is defined as the locus of the centers of curvature of

(c) The Author(s). 2019 Open Access This article is distributed under the terms of the Creative Commons Attribution 4.0 International License (http://creativecommons.org/licenses/by/4.0/), which permits unrestricted use, distribution, and reproduction in any medium, provided you give appropriate credit to the original author(s) and the source, provide a link to the Creative Commons license, and indicate if changes were made. 
the curve. The original curves are then defined as the involute of the evolute. The simplest case is that of a circle, which has only one center of curvature (its center), which is a degenerate evolute and the circle itself is the involute of this point.

Izumiya et al. defined the evolute curve in hyperbolic 2-space and found its equation. Following the works of them, we defined the evolute curve in hyperbolic 3-space and de Sitter 3-space and found its equations (see Definitions (2) and (3), and for more details, see $[1,6-10])$.

In this paper, we calculate the Frenet apparatus of the evolute curve in terms of the apparatus of its involute curve in hyperbolic 2-space, hyperbolic 3-space, and de Sitter 3 -space. We hope that our results can be seen as refinement and generalization of many corresponding results existing in the literature and useful in mathematical modeling and some other applications.

\section{Preliminaries}

In this section, we give the basic notions and familiar results in Lorentzian geometry which we need in this paper (for more details, see [7-12]).

\section{Hyperbolic 2-space}

Let $R^{3}=\left\{\left(x_{1}, x_{2}, x_{3}\right) \mid x_{1}, x_{2}, x_{3} \in R\right\}$ be a three-dimensional vector space and $x=$ $\left(x_{1}, x_{2}, x_{3}\right)$ and $y=\left(y_{1}, y_{2}, y_{3}\right)$ be two vectors in $R^{3}$. The pseudo-scalar product of $x$ and $y$ is defined by $\langle x, y\rangle=-x_{1} y_{1}+x_{2} y_{2}+x_{3} y_{3}$. $\left(R^{3},\langle\rangle,\right)$ is called a three-dimensional pseudoEuclidean space or Minkowski 3-space. We write $E_{1}^{3}$ instead of $\left(R^{3},\langle\rangle,\right)$. We say that a vector $x$ in $E_{1}^{3}$ is spacelike, lightlike, or timelike if $\langle x, x\rangle>0,\langle x, x\rangle=0$ or $\langle x, x\rangle<0$, respectively. We now define spheres in $E_{1}^{3}$ as follows:

$$
\left\{\begin{array}{l}
H_{+}^{2}=\left\{x \in E_{1}^{3} \mid-x_{1}^{2}+x_{2}^{2}+x_{3}^{2}=-1, x_{1} \geq 1\right\} \\
H_{-}^{2}=\left\{x \in E_{1}^{3} \mid-x_{1}^{2}+x_{2}^{2}+x_{3}^{2}=-1, x_{1} \leq-1\right\} \\
S_{1}^{2}=\left\{x \in E_{1}^{3} \mid-x_{1}^{2}+x_{2}^{2}+x_{3}^{2}=1\right\}
\end{array}\right.
$$

We call $H_{ \pm}^{2}$ a hyperbola and $S_{1}^{2}$ a pseudo-sphere. Now, we discuss some basic facts of curves in hyperbolic 2-space, which are needed in the sequel.

Let $\alpha: I \longrightarrow H_{+}^{2} \subset E_{1}^{3} ; \quad \alpha(t)=\left(x_{1}(t), x_{2}(t), x_{3}(t)\right)$ be a smooth regular curve in $H_{+}^{2}$ (i.e., $\alpha^{\prime}(t) \neq 0$ ) for any $t \in I$, where $I$ is an open interval. It is easy to show that $\left\langle\alpha^{\prime}(t), \alpha^{\prime}(t)\right\rangle>0$, for any $t \in I$. We call such a curve a spacelike curve. The norm of the vector $x \in E_{1}^{3}$ is defined by $\|x\|=\sqrt{|\langle x, x\rangle|}$. The arc-length of a spacelike curve $\alpha$, measured from $\alpha\left(t_{0}\right), t_{0} \in I$ is $s(t)=\int_{t_{0}}^{t}\left\|\alpha^{\prime}(t)\right\| d t$. Then, the parameter $s$ is determined such that $\|\dot{\alpha}(s)\|=1$, where $\dot{\alpha}(s)=\frac{d \alpha(s)}{d s}$. So, we say that a spacelike curve $\alpha$ is parameterized by arc-length, if it satisfies $\|\dot{\alpha}(s)\|=1$. Throughout the remainder in this paper, we denote the parameter $s$ of $\alpha$ as the arc-length parameter. Let us denote $\mathbf{T}(s)=\dot{\alpha}(s)$, and we call $\mathbf{T}(s)$ a unit tangent vector of $\alpha$ at $s$.

For any $x=\left(x_{1}, x_{2}, x_{3}\right), y=\left(y_{1}, y_{2}, y_{3}\right) \in E_{1}^{3}$, the pseudo-vector product of $x$ and $y$ is defined as follows:

$$
x \wedge y=\left|\begin{array}{ccc}
-e_{1} & e_{2} & e_{3} \\
x_{1} & x_{2} & x_{3} \\
y_{1} & y_{2} & y_{3}
\end{array}\right|=\left(-\left(x_{2} y_{3}-x_{3} y_{2}\right), x_{3} y_{1}-x_{1} y_{3}, x_{1} y_{2}-x_{2} y_{1}\right) .
$$


We remark that $\langle x \wedge y, z\rangle=\operatorname{det}(x y z)$. Hence, $x \wedge y$ is pseudo-orthogonal to $x, y$. We now set a vector $\mathbf{E}(s)=\alpha(s) \wedge \mathbf{T}(s)$. By definition, we can calculate that $\langle\mathbf{E}(s), \mathbf{E}(s)\rangle=1$ and $\langle\alpha(s), \alpha(s)\rangle=-1$. We can also show that $\mathbf{T}(s) \wedge \mathbf{E}(s)=-\alpha(s)$ and $\alpha(s) \wedge \mathbf{E}(s)=-\mathbf{T}(s)$. Therefore, we have a pseudo-orthonormal frame $\{\alpha(s), \mathbf{T}(s), \mathbf{E}(s)\}$ along $\alpha(s)$. We have the following hyperbolic Frenet-Serret formula of plane curves:

$$
\left\{\begin{array}{l}
\dot{\alpha}(s)=\mathbf{T}(s) \\
\dot{\mathbf{T}}(s)=\alpha(s)+\kappa_{g}(s) \mathbf{E}(s) \\
\dot{\mathbf{E}}(s)=-\kappa_{g}(s) \mathbf{T}(s),
\end{array}\right.
$$

or in the matrix form:

$$
\left[\begin{array}{c}
\dot{\alpha}(s) \\
\dot{\mathbf{T}}(s) \\
\dot{\mathbf{E}}(s)
\end{array}\right]=\left[\begin{array}{ccc}
0 & 1 & 0 \\
1 & 0 & \kappa_{g} \\
0 & -\kappa_{g} & 0
\end{array}\right]\left[\begin{array}{c}
\alpha(s) \\
\mathbf{T}(s) \\
\mathbf{E}(s)
\end{array}\right]
$$

where $\kappa_{g}$ is the geodesic curvature of the curve $\alpha$ in $H_{+}^{2}$, which is given by

$$
\kappa_{g}(s)=\operatorname{det}(\alpha(s) \mathbf{T}(s) \dot{\mathbf{T}}(s)) .
$$

\section{Hyperbolic 3-space}

Let $R^{4}$ be a four-dimensional vector space. For any $x=\left(x_{1}, x_{2}, x_{3}, x_{4}\right), y=\left(y_{1}, y_{2}, y_{3}, y_{4}\right) \in$ $R^{4}$, the pseudo-scalar product of $x$ and $y$ is defined by $\langle x, y\rangle=-x_{1} y_{1}+x_{2} y_{2}+x_{3} y_{3}+x_{4} y_{4}$. $\left(R^{4},\langle\rangle,\right)$ is called a Minkowski 4 -space and denoted by $E_{1}^{4}$. We say that a vector $x \in E_{1}^{4}$ is spacelike, lightlike, or timelike if $\left\langle x_{1}, x_{2}\right\rangle>0,\left\langle x_{1}, x_{2}\right\rangle=0$ or $\left\langle x_{1}, x_{2}\right\rangle<0$, respectively. The norm of the vector $x \in E_{1}^{4}$ is defiend by $\|x\|=\sqrt{|\langle x, x\rangle|}$. For a non-zero vector $v \in E_{1}^{4}$ and a real number $c$, we define a space with pseudo-normal $v$ by

$$
S(v, c)=\left\{x \in E_{1}^{4} \mid\langle x, v\rangle=c\right\} .
$$

The space $S(v, c)$ is called a spacelike space, a timelike space, or a lightlike space if $v$ is timelike, spacelike, or lightlike, respectively.

Now, we define a hyperbolic 3-space by

$$
H_{+}^{3}(-1)=\left\{x \in E_{1}^{4} \mid\langle x, x\rangle=-1, x_{1}>0\right\} .
$$

For any $x=\left(x_{1}, x_{2}, x_{3}, x_{4}\right), y=\left(y_{1}, y_{2}, y_{3}, y_{4}\right)$ and $z=\left(z_{1}, z_{2}, z_{3}, z_{4}\right) \in E_{1}^{4}$, the pseudovector product of $x, y$, and $z$ is defined as follows:

$$
\begin{aligned}
x \wedge y \wedge z & =\left|\begin{array}{llll}
-i & j & k & l \\
x_{1} & x_{2} & x_{3} & x_{4} \\
y_{1} & y_{2} & y_{3} & y_{4} \\
z_{1} & z_{2} & z_{3} & z_{4}
\end{array}\right| \\
& =\left(-\left|\begin{array}{lll}
x_{2} & x_{3} & x_{4} \\
y_{2} & y_{3} & y_{4} \\
z_{2} & z_{3} & z_{4}
\end{array}\right|,-\left|\begin{array}{lll}
x_{1} & x_{3} & x_{4} \\
y_{1} & y_{3} & y_{4} \\
z_{1} & z_{3} & z_{4}
\end{array}\right|,\left|\begin{array}{lll}
x_{1} & x_{2} & x_{4} \\
y_{1} & y_{2} & y_{4} \\
z_{1} & z_{2} & z_{4}
\end{array}\right|,-\left|\begin{array}{lll}
x_{1} & x_{2} & x_{3} \\
y_{1} & y_{2} & y_{3} \\
z_{1} & z_{2} & z_{3}
\end{array}\right|\right) .
\end{aligned}
$$

We now prepare some basic facts of curves in hyperbolic 3-space.

Let $\beta: I \longrightarrow H_{+}^{3} \subset E_{1}^{4} ; \quad \beta(t)=\left(x_{1}(t), x_{2}(t), x_{3}(t), x_{4}(t)\right)$ be a smooth regular curve in $H_{+}^{3}$ (i.e., $\beta^{\prime}(t) \neq 0$ ) for any $t \in I$ where $I$ is an open interval. So that $\left\langle\beta^{\prime}(t), \beta^{\prime}(t)\right\rangle>0$ for any $t \in I$. The arc-length of $\beta$ measured from $\beta\left(t_{\circ}\right), t_{\circ} \in I$ is $s(t)=\int_{t_{\circ}}^{t}\left\|\beta^{\prime}(t)\right\| d t$. Then, the parameter $s$ is determined such that $\|\dot{\beta}(s)\|=1$, where $\dot{\beta}(s)=\frac{d \beta(s)}{d s}$. So, we say that 
a spacelike curve $\beta$ is parameterized by arc-length if it satisfies that $\|\dot{\beta}(s)\|=1$. Let us denote $\mathbf{T}(s)=\dot{\beta}(s)$, and we call $\mathbf{T}(s)$ a unit tangent vector of $\beta$ at $s$.

Here, we construct the explicit differential geometry on curves in $H_{+}^{3}(-1)$. Let $\beta: I \longrightarrow$ $H_{+}^{3}(-1)$ be a regular curve. Since $H_{+}^{3}(-1)$ is a Riemannian manifold, we can reparameterize $\beta$ by the arc-length. Hence, we may assume that $\beta(s)$ is a unit speed curve. So, we have the tangent vector $\mathbf{T}(s)=\dot{\beta}(s)$ with $\|\mathbf{T}\|=1$. In case when $\langle\dot{\mathbf{T}}(s), \dot{\mathbf{T}}(s)\rangle \neq-1$, then we have a unit vector

$$
\mathbf{N}(s)=\frac{\dot{\mathbf{T}}(s)-\beta(s)}{\|\dot{\mathbf{T}}(s)-\beta(s)\|} .
$$

Moreover, define $\mathbf{E}(s)=\beta(s) \wedge \mathbf{T}(s) \wedge \mathbf{N}(s)$, then we have a pseudo-orthonormal frame $\{\beta(s), \mathbf{T}(s), \mathbf{N}(s), \mathbf{E}(s)\}$ of $E_{1}^{4}$ along $\beta$. By standard arguments, under the assumption that $\langle\dot{\mathbf{T}}(s), \dot{\mathbf{T}}(s)\rangle \neq-1$, we have the following Frenet formula:

$$
\left\{\begin{array}{l}
\dot{\beta}(s)=\mathbf{T}(s), \\
\dot{\mathbf{T}}(s)=\beta(s)+\kappa_{g} \mathbf{N}(s), \\
\dot{\mathbf{N}}(s)=-\kappa_{g} \mathbf{T}(s)+\tau_{g} \mathbf{E}(s), \\
\dot{\mathbf{E}}(s)=-\tau_{g} \mathbf{N}(s)
\end{array}\right.
$$

In another form:

$$
\left[\begin{array}{c}
\dot{\beta}(s) \\
\dot{\mathbf{T}}(s) \\
\dot{\mathbf{N}}(s) \\
\dot{\mathbf{E}}(s)
\end{array}\right]=\left[\begin{array}{cccc}
0 & 1 & 0 & 0 \\
1 & 0 & \kappa_{g} & 0 \\
0 & -\kappa_{g} & 0 & \tau_{g} \\
0 & 0 & -\tau_{g} & 0
\end{array}\right]\left[\begin{array}{c}
\beta(s) \\
\mathbf{T}(s) \\
\mathbf{N}(s) \\
\mathbf{E}(s)
\end{array}\right]
$$

where

$$
\begin{aligned}
\kappa_{g} & =\|\dot{\mathbf{T}}(s)-\beta(s)\|, \\
\tau_{g} & =-\frac{\operatorname{det}(\beta(s), \dot{\beta}(s), \ddot{\beta}(s), \dddot{\beta}(s))}{\left(\kappa_{g}(s)\right)^{2}}
\end{aligned}
$$

are the geodesic curvature and geodesic torsion of the curve $\beta$ in $H_{+}^{3}(-1)$, respectively.

$$
\text { Since }\langle\dot{\mathbf{T}}(s)-\beta(s), \dot{\mathbf{T}}(s)-\beta(s)\rangle=\langle\dot{\mathbf{T}}(s), \dot{\mathbf{T}}(s)\rangle+1 \text {, the condition }
$$

$$
\langle\dot{\mathbf{T}}(s), \dot{\mathbf{T}}(s)\rangle \neq-1,
$$

is equivalent to the condition $\kappa_{g}(s) \neq 0$. Moreover, we can show that the curve $\beta(s)$ satisfies the condition $\kappa_{g}(s) \equiv 0$ if and only if there exists a lightlike vector $c$ such that $\beta(s)-c$ is a geodesic. Such a curve is called an equidistant curve (see $[9,12])$.

\section{De Sitter 3-space}

Let $\gamma: I \longrightarrow S_{1}^{3}$ be a smooth and regular spacelike curve in $S_{1}^{3}$. We can parameterize it by arc-length $s$. Hence, we may assume that $\gamma(s)$ is a unit speed curve and we have the tangent vector $\mathbf{T}(s)=\dot{\gamma}(s)$ with $\|\mathbf{T}\|=1$. In this case, we call $\gamma$ a unit speed spacelike curve. If $\langle\dot{\mathbf{T}}(s), \dot{\mathbf{T}}(s)\rangle \neq 1$, then $\|\dot{\mathbf{T}}(s)+\gamma(s)\| \neq 0$, and we define the unit vector $\mathbf{N}(s)=\frac{\dot{\mathbf{T}}(s)+\gamma(s)}{\|\dot{\mathbf{T}}(s)+\gamma(s)\|}$. Moreover, define $\mathbf{E}(s)=\gamma(s) \wedge \mathbf{T}(s) \wedge \mathbf{N}(s)$, then we have a pseudoorthonormal frame $\{\gamma(s), \mathbf{T}(s), \mathbf{N}(s), \mathbf{E}(s)\}$ of $E_{1}^{4}$ along $\gamma$. By standard arguments, under the assumption that $\langle\dot{\mathbf{T}}(s), \dot{\mathbf{T}}(s)\rangle \neq 1$, we have the following Frenet-Serret type 
formula:

$$
\left\{\begin{array}{l}
\dot{\gamma}(s)=\mathbf{T}(s) \\
\dot{\mathbf{T}}(s)=-\gamma(s)+\kappa_{g} \mathbf{N}(s) \\
\dot{\mathbf{N}}(s)=-\delta(\gamma) \kappa_{g} \mathbf{T}(s)+\tau_{g} \mathbf{E}(s) \\
\dot{\mathbf{E}}(s)=\tau_{g} \mathbf{N}(s) .
\end{array}\right.
$$

It can be written as:

$$
\left[\begin{array}{c}
\dot{\gamma}(s) \\
\dot{\mathbf{T}}(s) \\
\dot{\mathbf{N}}(s) \\
\dot{\mathbf{E}}(s)
\end{array}\right]=\left[\begin{array}{cccc}
0 & 1 & 0 & 0 \\
-1 & 0 & \kappa_{g} & 0 \\
0 & -\delta(\gamma) \kappa_{g} & 0 & \tau_{g} \\
0 & 0 & \tau_{g} & 0
\end{array}\right]\left[\begin{array}{c}
\gamma(s) \\
\mathbf{T}(s) \\
\mathbf{N}(s) \\
\mathbf{E}(s)
\end{array}\right]
$$

where $\delta(\gamma)=\operatorname{sign}(\mathbf{N}(s)$ ) (which we shall write as simply $\delta$ ) and

$$
\left\{\begin{array}{l}
\kappa_{g}=\|\dot{\mathbf{T}}(s)+\gamma(s)\|, \\
\tau_{g}=\frac{\delta \operatorname{det}(\gamma(s), \dot{\gamma}(s), \ddot{\gamma}(s), \ddot{\gamma}(s))}{\left(\kappa_{g}(s)\right)^{2}},
\end{array}\right.
$$

are the geodesic curvature and geodesic torsion of the curve $\gamma$ in $S_{1}^{3}$, respectively.

Since $\langle\dot{\mathbf{T}}(s)+\gamma(s), \dot{\mathbf{T}}(s)+\gamma(s)\rangle=\langle\dot{\mathbf{T}}(s), \dot{\mathbf{T}}(s)\rangle-1$, the condition $\langle\dot{\mathbf{T}}(s), \dot{\mathbf{T}}(s)\rangle \neq 1$ is equivalent to the condition $\kappa_{g}(s) \neq 0$ (see [4]).

\section{The Frenet apparatus of an evolute curve in hyperbolic 2-space}

In this section, we introduce the Frenet apparatus of an evolute curve in terms of Frenet apparatus of its involute curve in $H_{+}^{2}$.

Definition 1 Let $\alpha: I \longrightarrow H_{+}^{2}$ be a smooth and regular spacelike curve in hyperbolic 2space. We define the hyperbolic evolute curve of $\alpha(s)$ under the assumption that $\kappa_{g}^{2}(s) \neq \pm 1$ in $H_{+}^{2}$ as;

$$
h_{\alpha}(s)=\frac{1}{\sqrt{\left|\kappa_{g}^{2}(s)-1\right|}}\left(\kappa_{g}(s) \alpha(s)+\mathbf{E}(s)\right) \text {. }
$$

We remark that $h_{\alpha}$ is located in $H_{+}^{2} \cup H_{-}^{2}$ if and only if $\kappa_{g}^{2}>1$. If $h_{\alpha}$ is located in $H_{-}^{2}$, we may consider $-h_{\alpha}(s)$ instead of $h_{\alpha}(s)$ and we call $\alpha(s)$ an involute curve of $h_{\alpha}(s)$ (for more details, see [10]).

We denote by the Frenet apparatus of an evolute curve $h_{\alpha}(s)$ by $\left\{h_{\alpha}(s), \mathbf{T}_{h_{\alpha}}(s), \mathbf{E}_{h_{\alpha}}(s), \mathcal{K}_{h_{\alpha}}(s)\right\}$.

Theorem 1 Let $\alpha(s): I \longrightarrow H_{+}^{2} \subset E_{1}^{3}$ be a unit speed spacelike involute curve in $H_{+}^{2}$, then the Frenet apparatus of the hyperbolic evolute curve $h_{\alpha}(s)$ are given as follows: 


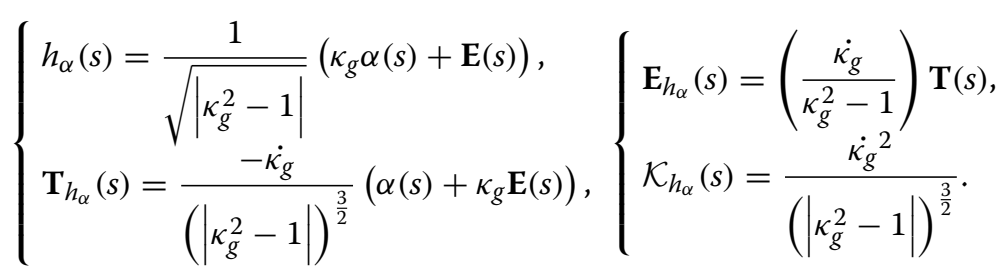

Proof From the definition of the evolute curve in hyperbolic 2-space, we can write

$$
h_{\alpha}(s)=\frac{1}{\sqrt{\left|\kappa_{g}^{2}-1\right|}}\left(\kappa_{g} \alpha(s)+\mathbf{E}(s)\right) \text {. }
$$

Differentiating both sides of the previous equation with respect to $s$ and substitute from Eq. (1), we obtain

$$
\mathbf{T}_{h_{\alpha}}(s)=\frac{-\kappa_{g} \dot{\kappa_{g}}}{\left(\left|\kappa_{g}^{2}-1\right|\right)^{\frac{3}{2}}}\left(\kappa_{g} \alpha+\mathbf{E}\right)+\frac{1}{\sqrt{\left|\kappa_{g}^{2}-1\right|}}\left(\dot{\kappa_{g}} \alpha+\kappa_{g} \mathbf{T}-\kappa_{g} \mathbf{T}\right),
$$

which can be written as

$$
\begin{aligned}
\mathbf{T}_{h_{\alpha}}(s) & =\frac{-\kappa_{g} \dot{\kappa_{g}}}{\left(\left|\kappa_{g}^{2}-1\right|\right)^{\frac{3}{2}}}\left(\kappa_{g} \alpha+\mathbf{E}\right)+\frac{\kappa_{g}^{2}-1}{\left(\left|\kappa_{g}^{2}-1\right|\right)^{\frac{3}{2}}}\left(\dot{\kappa_{g}} \alpha+\kappa_{g} \mathbf{T}-\kappa_{g} \mathbf{T}\right) \\
& =\frac{-\kappa_{g}^{2} \dot{\kappa_{g}}}{\left(\left|\kappa_{g}^{2}-1\right|\right)^{\frac{3}{2}}} \alpha-\frac{\kappa_{g} \dot{\kappa_{g}}}{\left(\left|\kappa_{g}^{2}-1\right|\right)^{\frac{3}{2}}} \mathbf{E}+\frac{\kappa_{g}^{2} \dot{\kappa_{g}}}{\left(\left|\kappa_{g}^{2}-1\right|\right)^{\frac{3}{2}}} \alpha-\frac{\dot{\kappa_{g}}}{\left(\left|\kappa_{g}^{2}-1\right|\right)^{\frac{3}{2}}} \alpha,
\end{aligned}
$$

then, we get

$$
\mathbf{T}_{h_{\alpha}}(s)=\frac{-\dot{\kappa_{g}}}{\left(\left|\kappa_{g}^{2}-1\right|\right)^{\frac{3}{2}}} \alpha(s)-\frac{\kappa_{g} \dot{\kappa_{g}}}{\left(\left|\kappa_{g}^{2}-1\right|\right)^{\frac{3}{2}}} \mathbf{E}(s) .
$$

Since $\mathbf{E}_{h_{\alpha}}(s)=h_{\alpha}(s) \wedge \mathbf{T}_{h_{\alpha}}(s)$, we have

$$
\mathbf{E}_{h_{\alpha}}(s)=\left|\begin{array}{ccc}
-\alpha(s) & \mathbf{T}(s) & \mathbf{E}(s) \\
\frac{\kappa_{g}}{\sqrt{\left|\kappa_{g}^{2}-1\right|}} & 0 & \frac{1}{\sqrt{\left|\kappa_{g}^{2}-1\right|}} \\
\frac{-\dot{\kappa_{g}}}{\left(\left|\kappa_{g}^{2}-1\right|\right)^{\frac{3}{2}}} & 0 & \frac{-\kappa_{g} \dot{\kappa_{g}}}{\left(\left|\kappa_{g}^{2}-1\right|\right)^{\frac{3}{2}}}
\end{array}\right|
$$

then, we get

$$
\mathbf{E}_{h_{\alpha}}(s)=\left(\frac{\dot{\kappa_{g}}}{\kappa_{g}^{2}-1}\right) \mathbf{T}(s)
$$

Also, by differentiating the Eq. (9) and substitute from Eq. (1), we obtain 


$$
\dot{\mathbf{T}}_{h_{\alpha}}(s)=\left\{\begin{array}{l}
\left(\frac{\ddot{\kappa_{g}}-\kappa_{g}^{2} \ddot{\kappa_{g}}+3 \kappa_{g} \dot{\kappa_{g}}}{\left(\left|\kappa_{g}^{2}-1\right|\right)^{\frac{5}{2}}}\right) \alpha(s)+\frac{\dot{\kappa_{g}}}{\sqrt{\left|\kappa_{g}^{2}-1\right|}} \mathbf{T}(s) \\
+\left(\frac{\kappa_{g} \ddot{\kappa}_{g}-\kappa_{g}^{3} \ddot{\kappa}_{g}+3 \kappa_{g}^{2} \dot{\kappa}_{g}-\kappa_{g}^{2}{\dot{\kappa_{g}}}^{2}+\dot{\kappa}_{g}^{2}}{\left(\left|\kappa_{g}^{2}-1\right|\right)^{\frac{5}{2}}}\right) \mathbf{E}(s) .
\end{array}\right.
$$

Because $\mathcal{K}_{h_{\alpha}}=\operatorname{det}\left(h_{\alpha}(s) \mathbf{T}_{h_{\alpha}} \dot{\mathbf{T}}_{h_{\alpha}}\right)$, then from Eqs. (8-10), we get

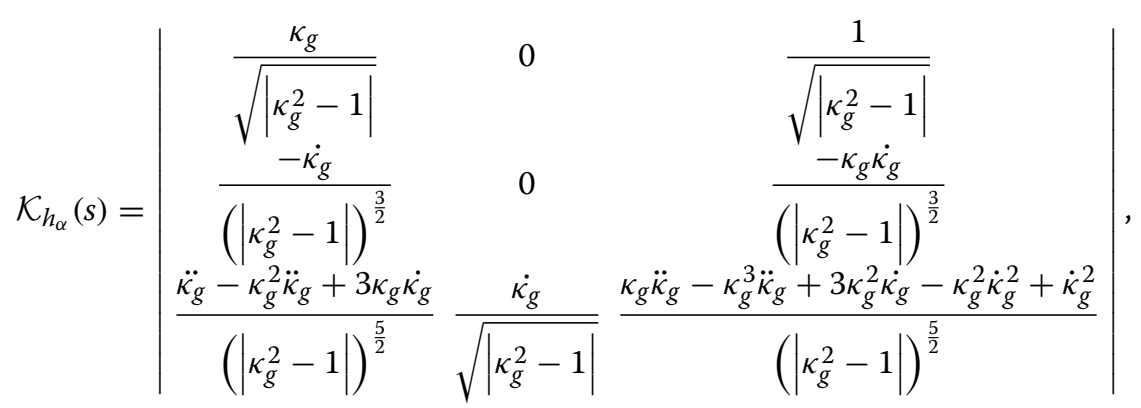

which can be written as:

$$
\begin{aligned}
\mathcal{K}_{h_{\alpha}}= & \left(\frac{\kappa_{g}}{\sqrt{\left|\kappa_{g}^{2}-1\right|}}\right)\left(\frac{\dot{\kappa_{g}}}{\sqrt{\left|\kappa_{g}^{2}-1\right|}}\right)\left(\frac{\kappa_{g} \dot{\kappa_{g}}}{\left(\left|\kappa_{g}^{2}-1\right|\right)^{\frac{3}{2}}}\right) \\
& -\left(\frac{1}{\sqrt{\left|\kappa_{g}^{2}-1\right|}}\right)\left(\frac{\dot{\kappa_{g}}}{\left(\left|\kappa_{g}^{2}-1\right|\right)^{\frac{3}{2}}}\right)\left(\frac{\dot{\kappa_{g}}}{\sqrt{\left|\kappa_{g}^{2}-1\right|}}\right) \\
= & \left(\frac{\kappa_{g}^{2} \dot{\kappa}_{g}^{2}}{\left(\left|\kappa_{g}^{2}-1\right|\right)^{\frac{5}{2}}}\right)-\left(\frac{\dot{\kappa_{g}}}{\left(\left|\kappa_{g}^{2}-1\right|\right)^{\frac{5}{2}}}\right) \\
= & \left(\frac{\dot{\dot{\kappa}_{g}}{ }^{2}\left(\kappa_{g}^{2}-1\right)}{\left(\left|\kappa_{g}^{2}-1\right|\right)^{\frac{5}{2}}}\right) .
\end{aligned}
$$

In a simple form

$$
\mathcal{K}_{h_{\alpha}}=\frac{{\dot{\kappa_{g}}}^{2}}{\left(\left|\kappa_{g}^{2}-1\right|\right)^{\frac{3}{2}}} .
$$

In the light of the above calculations, the proof is completed.

\section{The Frenet apparatus of an evolute curve in hyperbolic 3-space}

Here, as in the case of hyperbolic 2-space, we construct the Frenet apparatus of an evolute curve using Frenet apparatus of its involute curve in the hyperbolic 3-space $H_{+}^{3}(-1)$ and define its equation. We start as follows: 
Definition 2 For a given involute curve $\beta$ in $H_{+}^{3}(-1)$, the hyperbolic evolute curve $h_{\beta}$ : $I \longrightarrow H_{+}^{3}$ of $\beta(s)$ is defined by

$$
h_{\beta}(s)=\frac{1}{\sqrt{\left|\kappa_{g}^{2}-\left(\frac{\dot{\kappa_{g}}}{\kappa_{g} \tau_{g}}\right)^{2}-1\right|}}\left(\kappa_{g} \beta(s)+\mathbf{N}(s)-\frac{\dot{\kappa_{g}}}{\kappa_{g} \tau_{g}} \mathbf{E}(s)\right),
$$

under the assumption that $\kappa_{g}^{2}-\left(\frac{\dot{\kappa_{g}}}{\kappa_{g} \tau_{g}}\right)^{2}>1$.

We remark that $h_{\beta}(s)$ is located in $H_{+}^{3}(-1)$ if and only if $\kappa_{g}^{2}-\left(\frac{\dot{\kappa_{g}}}{\kappa_{g} \tau_{g}}\right)^{2}>1$, where $\beta(s)$ is an involute curve of $h_{\beta}(s)$ (see [6]).

Now, we denote by $\left\{h_{\beta}(s), \mathbf{T}_{h_{\beta}}(s), \mathbf{N}_{h_{\beta}}(s), \mathbf{E}_{h_{\beta}}(s), \mathcal{K}_{h_{\beta}}(s), \mathcal{T}_{h_{\beta}}(s)\right\}$ the Frenet apparatus of $h_{\beta}(s)$ and we formulate the following theorem.

Theorem 2 Let $h_{\beta}(s)$ be a unit speed spacelike evolute curve of $\beta(s)$, then the Frenet apparatus of $h_{\beta}(s)$ can be expressed as:

$$
\begin{aligned}
\mathbf{T}_{h_{\beta}}(s)= & \dot{\mu}_{1} \beta(s)+\left(\mu_{1}-\kappa_{g} \mu_{2}\right) \mathbf{T}(s)+\left(\dot{\mu}_{2}-\tau_{g} \mu_{3}\right) \mathbf{N}(s)+\left(\dot{\mu}_{3}+\tau_{g} \mu_{2}\right) \mathbf{E}(s), \\
\mathbf{N}_{h_{\beta}}(s)= & \left(\left|-\left(\eta_{1}-\mu_{1}\right)^{2}+\eta_{2}^{2}+\left(\eta_{3}-\mu_{2}\right)^{2}+\left(\eta_{4}-\mu_{3}\right)^{2}\right|\right)^{\frac{-1}{2}}\left(\left(\left(\eta_{1}-\mu_{1}\right) \beta(s)\right.\right. \\
& \left.\left.+\eta_{2} \mathbf{T}(s)+\left(\eta_{3}-\mu_{2}\right) \mathbf{N}(s)+\left(\eta_{4}-\mu_{3}\right) \mathbf{E}(s)\right)\right), \\
\mathcal{K}_{h_{\beta}}= & \sqrt{\left|-\left(\eta_{1}-\mu_{1}\right)^{2}+\eta_{2}^{2}+\left(\eta_{3}-\mu_{2}\right)^{2}+\left(\eta_{4}-\mu_{3}\right)^{2}\right|}, \\
\mathbf{E}_{h_{\beta}}(s)= & -\frac{1}{\mathcal{K}_{\beta}}\left(\left(-\mu_{2}\left(\left(\mu_{1}-\kappa_{g} \mu_{2}\right)\left(\eta_{4}-\mu_{3}\right)-\eta_{2}\left(\dot{\mu}_{2}-\tau_{g} \mu_{3}\right)\right)+\mu_{3}\left(\left(\mu_{1}-\kappa_{g} \mu_{2}\right)\left(\eta_{3}-\mu_{2}\right)\right.\right.\right. \\
& \left.\left.-\eta_{2}\left(\dot{\mu}_{2}-\tau_{g} \mu_{3}\right)\right)\right) \beta(s)+\left(\mu_{1}\left(\left(\dot{\mu}_{2}-\tau_{g} \mu_{3}\right)\left(\eta_{4}-\mu_{3}\right)-\left(\eta_{3}-\mu_{2}\right)\left(\dot{\mu}_{3}+\tau_{g} \mu_{2}\right)\right)\right. \\
& \left.-\mu_{2}\left(\dot{\mu}_{1}\left(\eta_{4}-\mu_{3}\right)-\left(\eta_{1}-\mu_{1}\right)\left(\dot{\mu}_{3}+\tau_{g} \mu_{2}\right)\right)+\mu_{3}\left(\dot{\mu}_{1}\left(\eta_{3}-\mu_{2}\right)-\left(\eta_{1}-\mu_{1}\right)\left(\dot{\mu}_{2}-\tau_{g} \mu_{3}\right)\right)\right) \mathbf{T}(s) \\
& -\left(\mu_{1}\left(\left(\mu_{1}-\kappa_{g} \mu_{2}\right)\left(\eta_{4}-\mu_{3}\right)-\eta_{2}\left(\dot{\mu}_{3}+\tau_{g} \mu_{2}\right)\right)+\mu_{3}\left(\dot{\mu}_{1} \eta_{2}-\left(\eta_{1}-\mu_{1}\right)\left(\mu_{1}-\kappa_{g} \mu_{2}\right)\right)\right) \mathbf{N}(s) \\
& \left.+\left(\mu_{1}\left(\left(\mu_{1}-\kappa_{g} \mu_{2}\right)\left(\eta_{3}-\mu_{2}\right)-\eta_{2}\left(\dot{\mu}_{2}+\tau_{g} \mu_{3}\right)\right)+\mu_{2}\left(\dot{\mu}_{1} \eta_{2}-\left(\eta_{1}-\mu_{1}\right)\left(\mu_{1}-\kappa_{g} \mu_{2}\right)\right)\right) \mathbf{E}(s)\right), \\
& 1 \\
\mathcal{T}_{h_{\beta}}(s)= & \frac{1}{\mathcal{K}_{\beta}^{2}}\left(\mu_{1}\left(\left(\mu_{1}-\kappa_{g} \mu_{2}\right)\left(\eta_{3} \zeta_{4}-\eta_{4} \zeta_{3}\right)-\left(\dot{\mu}_{2}-\tau_{g} \mu_{3}\right)\left(\eta_{2} \zeta_{4}-\eta_{4} \zeta_{2}\right)+\left(\dot{\mu}_{3}-\tau_{g} \mu_{2}\right)\left(\eta_{2} \zeta_{3}-\eta_{3} \zeta_{2}\right)\right)\right. \\
& -\mu_{2}\left(\dot{\mu}_{1}\left(\eta_{2} \zeta_{4}-\eta_{4} \zeta_{2}\right)-\left(\mu_{1}-\kappa_{g} \mu_{2}\right)\left(\eta_{1} \zeta_{4}-\eta_{4} \zeta_{1}\right)+\left(\dot{\mu}_{3}-\tau_{g} \mu_{2}\right)\left(\eta_{1} \zeta_{2}-\eta_{2} \zeta_{1}\right)\right) \\
& \left.+\mu_{3}\left(\dot{\mu}_{1}\left(\eta_{2} \zeta_{3}-\eta_{3} \zeta_{2}\right)-\left(\mu_{1}-\kappa_{g} \mu_{2}\right)\left(\eta_{1} \zeta_{3}-\eta_{3} \zeta_{1}\right)+\left(\dot{\mu}_{2}-\tau_{g} \mu_{3}\right)\left(\eta_{1} \zeta_{2}-\eta_{2} \zeta_{1}\right)\right)\right),
\end{aligned}
$$

where $\mu_{1}, \mu_{2}, \mu_{3}, \eta_{1}, \eta_{2}, \eta_{3}, \eta_{4}, \zeta_{1}, \zeta_{2}, \zeta_{3}$, and $\zeta_{4}$ are smooth functions.

Proof According to the Definition (2), we have

$$
h_{\beta}(s)=\frac{\kappa_{g}}{|| \kappa_{g}^{2}-\left(\frac{\dot{\kappa_{g}}}{\kappa_{g} \tau_{g}}\right)^{2}-1 \mid} \beta+\frac{1}{\sqrt{\left|\kappa_{g}^{2}-\left(\frac{\dot{\kappa_{g}}}{\kappa_{g} \tau_{g}}\right)^{2}-1\right|}} \mathbf{N}-\frac{\dot{\kappa_{g}}}{\kappa_{g} \tau_{g}|| \kappa_{g}^{2}-\left(\frac{\dot{\kappa_{g}}}{\kappa_{g} \tau_{g}}\right)^{2}-1 \mid} \mathbf{E} .
$$

If we denote 


$$
\begin{aligned}
& \mu_{1}=\frac{\kappa_{g}}{\sqrt{\left|\kappa_{g}^{2}-\left(\frac{\dot{\kappa_{g}}}{\kappa_{g} \tau_{g}}\right)^{2}-1\right|}}, \mu_{2}=\frac{1}{\sqrt{\left|\kappa_{g}^{2}-\left(\frac{\dot{\kappa_{g}}}{\kappa_{g} \tau_{g}}\right)^{2}-1\right|}}, \\
& \mu_{3}=-\frac{\dot{\kappa}_{g} \tau_{g} \sqrt{\left|\kappa_{g}^{2}-\left(\frac{\dot{\kappa_{g}}}{\kappa_{g} \tau_{g}}\right)^{2}-1\right|}}{\sqrt{\left|{ }^{2}\right|}},
\end{aligned}
$$

then, the evolute curve $h_{\beta}(s)$ can take the form

$$
h_{\beta}(s)=\mu_{1} \beta(s)+\mu_{2} \mathbf{N}(s)+\mu_{3} \mathbf{E}(s) .
$$

Differentiating both sides of the previous equation with respect to $s$ and substitute from Eq. (3), we obtain

$$
\begin{aligned}
\mathbf{T}_{h_{\beta}}(s)= & \dot{\mu}_{1} \beta(s)+\mu_{1} \dot{\beta}(s)+\dot{\mu}_{2} \mathbf{N}(s)+\mu_{2} \dot{\mathbf{N}}(s)+\dot{\mu}_{3} \mathbf{E}(s)+\mu_{3} \dot{\mathbf{E}}(s) \\
= & \dot{\mu}_{1} \beta(s)+\mu_{1} \mathbf{T}(s)+\dot{\mu}_{2} \mathbf{N}(s)+\mu_{2}\left(-\kappa_{g} \mathbf{T}(s)+\tau_{g} \mathbf{E}(s)\right) \\
& +\dot{\mu}_{3} \mathbf{E}(s)-\mu_{3}\left(\tau_{g} \mathbf{N}(s)\right) \\
= & \dot{\mu}_{1} \beta(s)+\left(\mu_{1}-\kappa_{g} \mu_{2}\right) \mathbf{T}(s)+\left(\dot{\mu}_{2}-\tau_{g} \mu_{3}\right) \mathbf{N}(s) \\
& +\left(\dot{\mu}_{3}+\tau_{g} \mu_{2}\right) \mathbf{E}(s) .
\end{aligned}
$$

Following the differentiating of Eq. (13), we get

$$
\dot{\mathbf{T}}_{h_{\beta}}(s)=\left\{\begin{array}{l}
\left(\ddot{\mu}_{1}+\mu_{1}-\kappa_{g} \mu_{2}\right) \beta(s)+\left(2 \dot{\mu}_{1}-\dot{\kappa_{g}} \mu_{2}-2 \kappa_{g} \dot{\mu}_{2}+\kappa_{g} \tau_{g} \mu_{3}\right) \mathbf{T}(s) \\
+\left(\kappa_{g} \mu_{1}-\kappa_{g}^{2} \mu_{2}+\ddot{\mu}_{2}-\dot{\tau}_{g} \mu_{3}-2 \tau_{g} \dot{\mu}_{3}-\tau_{g}^{2} \mu_{2}\right) \mathbf{N}(s) \\
+\left(\tau_{g} \dot{\mu}_{2}-\tau_{g}^{2} \mu_{3}+\ddot{\mu}_{3}+\dot{\tau}_{g} \mu_{2}+\tau_{g} \dot{\mu}_{2}\right) \mathbf{E}(s),
\end{array}\right.
$$

which can be written in a simple form

$$
\dot{\mathbf{T}}_{h_{\beta}}(s)=\eta_{1} \beta(s)+\eta_{2} \mathbf{T}(s)+\eta_{3} \mathbf{N}(s)+\eta_{4} \mathbf{E}(s),
$$

where

$$
\left\{\begin{array}{l}
\eta_{1}=\left(\ddot{\mu}_{1}+\mu_{1}-\kappa_{g} \mu_{2}\right) \\
\eta_{2}=\left(2 \dot{\mu}_{1}-\dot{\kappa}_{g} \mu_{2}-2 \kappa_{g} \dot{\mu}_{2}+\kappa_{g} \tau_{g} \mu_{3}\right) \\
\eta_{3}=\left(\kappa_{g} \mu_{1}-\kappa_{g}^{2} \mu_{2}+\ddot{\mu}_{2}-\dot{\tau}_{g} \mu_{3}-2 \tau_{g} \dot{\mu}_{3}-\tau_{g}^{2} \mu_{2}\right) \\
\eta_{4}=\left(\tau_{g} \dot{\mu}_{2}-\tau_{g}^{2} \mu_{3}+\ddot{\mu}_{3}+\dot{\tau}_{g} \mu_{2}+\tau_{g} \dot{\mu}_{2}\right) .
\end{array}\right.
$$

Thus, from Eqs. (12) and (14), we obtain

$$
\mathbf{N}_{h_{\beta}}(s)=\frac{\dot{\mathbf{T}}_{\beta}(s)-h_{\beta}(s)}{\left\|\dot{\mathbf{T}}_{\beta}(s)-h_{\beta}(s)\right\|},
$$

Following the above, we can write

$$
\begin{aligned}
\mathbf{N}_{h_{\beta}}(s)= & \left(\left|-\left(\eta_{1}-\mu_{1}\right)^{2}+\eta_{2}^{2}+\left(\eta_{3}-\mu_{2}\right)^{2}+\left(\eta_{4}-\mu_{3}\right)^{2}\right|\right)^{\frac{-1}{2}}\left(\left(\left(\eta_{1}-\mu_{1}\right) \beta(s)\right.\right. \\
& \left.\left.+\eta_{2} \mathbf{T}(s)+\left(\eta_{3}-\mu_{2}\right) \mathbf{N}(s)+\left(\eta_{4}-\mu_{3}\right) \mathbf{E}(s)\right)\right) .
\end{aligned}
$$

Also, from Eqs. (4) and (17), we obtain

$$
\mathcal{K}_{h_{\beta}}=\sqrt{\left|-\left(\eta_{1}-\mu_{1}\right)^{2}+\eta_{2}^{2}+\left(\eta_{3}-\mu_{2}\right)^{2}+\left(\eta_{4}-\mu_{3}\right)^{2}\right|} .
$$

Therefore, by differentiating (14) and using (3), we can obtain 


$$
\begin{aligned}
{\dddot{h_{\beta}}}_{\beta}(s)= & \left(\ddot{\mu}_{1}+3 \dot{\mu}_{1}-2 \dot{\kappa}_{g} \mu_{2}-3 \kappa_{g} \dot{\mu}_{2}+\kappa_{g} \tau_{g} \mu_{3}\right) \beta(s)+\left(3 \ddot{\mu}_{1}+\mu_{1}-\kappa_{g} \mu_{2}-\ddot{\kappa}_{g} \mu_{2}\right. \\
& \left.-3 \dot{\kappa_{g}} \dot{\mu}_{2}-3 \kappa_{g} \ddot{\mu}_{2}+\dot{\kappa_{g}} \tau_{g} \mu_{3}+2 \kappa_{g} \dot{\tau}_{g} \mu_{3}+3 \kappa_{g} \tau_{g} \dot{\mu}_{3}\right) \mathbf{T}(s)+\left(3 \kappa_{g} \dot{\mu}_{1}-3 \kappa_{g} \dot{\kappa}_{g} \mu_{2}\right. \\
& -3 \kappa_{g}^{2} \dot{\mu}_{2}+\dot{\kappa_{g}} \mu_{1}+\kappa_{g}^{2} \tau_{g} \mu_{3}-3 \tau_{g} \dot{\tau}_{g} \mu_{2}-2 \tau_{g}^{2} \dot{\mu}_{2}+\dddot{\mu}_{2}-\ddot{\tau}_{g} \mu_{3}-3 \dot{\tau}_{g} \dot{\mu}_{3}-3 \tau_{g} \ddot{\mu}_{3} \\
& \left.-\tau_{g}^{2} \dot{\mu}_{2}-\tau_{g}^{2} \mu_{3}\right) \mathbf{N}(s)+\left(\kappa_{g} \tau_{g} \mu_{1}-\kappa_{g}^{2} \tau_{g} \mu_{2}-\tau_{g}^{3} \mu_{2}+3 \tau_{g} \ddot{\mu}_{2}-3 \tau_{g} \dot{\tau}_{g} \mu_{3}+\dddot{\mu}_{3}\right. \\
& \left.-3 \tau_{g}^{2} \dot{\mu}_{3}+3 \dot{\tau}_{g} \dot{\mu}_{2}+\ddot{\tau}_{g} \mu_{2}\right) \mathbf{E}(s),
\end{aligned}
$$

which can be written as:

$$
\dddot{h}_{\beta}(s)=\zeta_{1} \beta(s)+\zeta_{2} \mathbf{T}(s)+\zeta_{3} \mathbf{N}(s)+\zeta_{4} \mathbf{E}(s) \text {, }
$$

Noting that

$$
\left\{\begin{aligned}
\zeta_{1}= & \left(\ddot{\mu}_{1}+3 \dot{\mu}_{1}-2 \dot{\kappa}_{g} \mu_{2}-3 \kappa_{g} \dot{\mu}_{2}+\kappa_{g} \tau_{g} \mu_{3}\right) \\
\zeta_{2}= & \left(3 \ddot{\mu}_{1}+\mu_{1}-\kappa_{g} \mu_{2}-\ddot{\kappa}_{g} \mu_{2}-3 \dot{\kappa}_{g} \dot{\mu}_{2}-3 \kappa_{g} \ddot{\mu}_{2}\right. \\
& \left.+\dot{\kappa}_{g} \tau_{g} \mu_{3}+2 \kappa_{g} \dot{\tau}_{g} \mu_{3}+3 \kappa_{g} \tau_{g} \dot{\mu}_{3}\right) \\
\zeta_{3}= & \left(3 \kappa_{g} \dot{\mu}_{1}-3 \kappa_{g} \dot{\kappa}_{g} \mu_{2}-3 \kappa_{g}^{2} \dot{\mu}_{2}+\dot{\kappa}_{g} \mu_{1}+\kappa_{g}^{2} \tau_{g} \mu_{3}\right. \\
& -3 \tau_{g} \dot{\tau}_{g} \mu_{2}-2 \tau_{g}^{2} \dot{\mu}_{2}+\ddot{\mu}_{2}-\ddot{\tau}_{g} \mu_{3}-3 \dot{\tau}_{g} \dot{\mu}_{3}-3 \tau_{g} \ddot{\mu}_{3} \\
& \left.-\tau_{g}^{2} \dot{\mu}_{2}-\tau_{g}^{2} \mu_{3}\right) \\
\zeta_{4}= & \left(\kappa_{g} \tau_{g} \mu_{1}-\kappa_{g}^{2} \tau_{g} \mu_{2}-\tau_{g}^{3} \mu_{2}+3 \tau_{g} \ddot{\mu}_{2}-3 \tau_{g} \dot{\tau}_{g} \mu_{3}\right. \\
& \left.+\dddot{\mu}_{3}-3 \tau_{g}^{2} \dot{\mu}_{3}+3 \dot{\tau}_{g} \dot{\mu}_{2}+\ddot{\tau}_{g} \mu_{2}\right) .
\end{aligned}\right.
$$

From Eqs. (4), (12), (13), (15), and (19), the torsion $\mathcal{T}_{\beta}$ is given by

$$
\mathcal{T}_{h_{\beta}}(s)=-\frac{1}{\mathcal{K}_{h_{\beta}}^{2}}\left|\begin{array}{cccc}
\mu_{1} & 0 & \mu_{2} & \mu_{3} \\
\dot{\mu}_{1} & \left(\mu_{1}-\kappa_{g} \mu_{2}\right) & \left(\dot{\mu}_{2}-\tau_{g} \mu_{3}\right) & \left(\dot{\mu}_{3}+\tau_{g} \mu_{2}\right) \\
\eta_{1} & \eta_{2} & \eta_{3} & \eta_{4} \\
\zeta_{1} & \zeta_{2} & \zeta_{3} & \zeta_{4}
\end{array}\right|,
$$

or

$$
\begin{aligned}
\mathcal{T}_{h_{\beta}}(s)= & \frac{1}{\mathcal{K}_{h_{\beta}}^{2}}\left(\mu_{1}\left(\left(\mu_{1}-\kappa_{g} \mu_{2}\right)\left(\eta_{3} \zeta_{4}-\eta_{4} \zeta_{3}\right)-\left(\dot{\mu}_{2}-\tau_{g} \mu_{3}\right)\left(\eta_{2} \zeta_{4}-\eta_{4} \zeta_{2}\right)+\left(\dot{\mu}_{3}-\tau_{g} \mu_{2}\right)\left(\eta_{2} \zeta_{3}-\eta_{3} \zeta_{2}\right)\right)\right. \\
& -\mu_{2}\left(\dot{\mu}_{1}\left(\eta_{2} \zeta_{4}-\eta_{4} \zeta_{2}\right)-\left(\mu_{1}-\kappa_{g} \mu_{2}\right)\left(\eta_{1} \zeta_{4}-\eta_{4} \zeta_{1}\right)+\left(\dot{\mu}_{3}-\tau_{g} \mu_{2}\right)\left(\eta_{1} \zeta_{2}-\eta_{2} \zeta_{1}\right)\right) \\
& \left.+\mu_{3}\left(\dot{\mu}_{1}\left(\eta_{2} \zeta_{3}-\eta_{3} \zeta_{2}\right)-\left(\mu_{1}-\kappa_{g} \mu_{2}\right)\left(\eta_{1} \zeta_{3}-\eta_{3} \zeta_{1}\right)+\left(\dot{\mu}_{2}-\tau_{g} \mu_{3}\right)\left(\eta_{1} \zeta_{2}-\eta_{2} \zeta_{1}\right)\right)\right) .
\end{aligned}
$$

In addition, from (12), (13), and (17), the vector $\mathbf{E}_{h_{\beta}}(s)=h_{\beta}(s) \wedge \mathbf{T}_{h_{\beta}}(s) \wedge \mathbf{N}_{h_{\beta}}(s)$ is computed as:

$$
\mathbf{E}_{h_{\beta}}(s)=\frac{1}{\mathcal{K}_{h_{\beta}}}\left|\begin{array}{cccc}
-\beta(s) & \mathbf{T}(s) & \mathbf{N}(s) & \mathbf{E}(s) \\
\mu_{1} & 0 & \mu_{2} & \mu_{3} \\
\dot{\mu}_{1} & \left(\mu_{1}-\kappa_{g} \mu_{2}\right) & \left(\dot{\mu}_{2}-\tau_{g} \mu_{3}\right) & \left(\dot{\mu}_{3}+\tau_{g} \mu_{2}\right) \\
\left(\eta_{1}-\mu_{1}\right) & \eta_{2} & \left(\eta_{3}-\mu_{2}\right) & \left(\eta_{4}-\mu_{3}\right)
\end{array}\right|,
$$

which can be taken in the form

$$
\begin{array}{r}
\mathbf{E}_{h_{\beta}}(s)=-\frac{1}{\mathcal{K}_{h_{\beta}}}\left|\begin{array}{ccc}
0 & \mu_{2} & \mu_{3} \\
\left(\mu_{1}-\kappa_{g} \mu_{2}\right) & \left(\dot{\mu}_{2}-\tau_{g} \mu_{3}\right) & \left(\dot{\mu}_{3}+\tau_{g} \mu_{2}\right) \\
\eta_{2} & \left(\eta_{3}-\mu_{2}\right) & \left(\eta_{4}-\mu_{3}\right)
\end{array}\right| \beta(s) \\
-\frac{1}{\mathcal{K}_{h_{\beta}}}\left|\begin{array}{ccc}
\mu_{1} & \mu_{2} & \mu_{3} \\
\dot{\mu}_{1} & \left(\dot{\mu}_{2}-\tau_{g} \mu_{3}\right) & \left(\dot{\mu}_{3}+\tau_{g} \mu_{2}\right) \\
\left(\eta_{1}-\mu_{1}\right) & \left(\eta_{3}-\mu_{2}\right) & \left(\eta_{4}-\mu_{3}\right)
\end{array}\right| \mathbf{T}(s)
\end{array}
$$




$$
\begin{gathered}
+\frac{1}{\mathcal{K}_{h_{\beta}}}\left|\begin{array}{ccc}
\mu_{1} & 0 & \mu_{3} \\
\dot{\mu}_{1} & \left(\mu_{1}-\kappa_{g} \mu_{2}\right) & \left(\dot{\mu}_{3}+\tau_{g} \mu_{2}\right) \\
\left(\eta_{1}-\mu_{1}\right) & \eta_{2} & \left(\eta_{4}-\mu_{3}\right)
\end{array}\right| \mathbf{N}(s) \\
-\frac{1}{\mathcal{K}_{h_{\beta}}}\left|\begin{array}{ccc}
\mu_{1} & 0 & \mu_{2} \\
\dot{\mu}_{1} & \left(\mu_{1}-\kappa_{g} \mu_{2}\right) & \left(\dot{\mu}_{2}-\tau_{g} \mu_{3}\right) \\
\left(\eta_{1}-\mu_{1}\right) & \eta_{2} & \left(\eta_{3}-\mu_{2}\right)
\end{array}\right| \mathbf{E}(s),
\end{gathered}
$$

In the light of the previous computations, the proof is completed.

\section{The Frenet apparatus of an evolute curve in de Sitter 3-space}

Similar to the case of the hyperbolic evolute curve, we introduce the definition Frenet apparatus of an evolute curve in the three-dimensional de Sitter space.

Definition 3 Let $\gamma$ be an involute curve in $S_{1}^{3}$, then the de Sitter evolute curve $d_{\gamma}: I \longrightarrow$ $S_{1}^{3}$ of $\gamma(s)$ is expressed as

$$
d_{\gamma}(s)=\frac{1}{\sqrt{\left|\left(\frac{\dot{\kappa_{g}}}{\kappa_{g} \tau_{g}}\right)^{2}-\kappa_{g}^{2}+1\right|}}\left(\kappa_{g} \gamma(s)+\mathbf{N}(s)+\frac{\dot{\kappa_{g}}}{\kappa_{g} \tau_{g}} \mathbf{E}(s)\right)
$$

under the assumption that $\kappa_{g}^{2}-\left(\frac{\dot{\kappa_{g}}}{\kappa_{g} \tau_{g}}\right)^{2}<1$.

We remark that $d_{\gamma}(s)$ is located in $S_{1}^{3}$ if and only if $\kappa_{g}^{2}-\left(\frac{\dot{\kappa_{g}}}{\kappa_{g} \tau_{g}}\right)^{2}<1$, where $\gamma(s)$ is an involute curve of $d_{\gamma}$ (see [1]).

We refer to $\left\{d_{\gamma}(s), \mathbf{T}_{d_{\gamma}}(s), \mathbf{N}_{d_{\gamma}}(s), \mathbf{E}_{d_{\gamma}}(s), \mathcal{K}_{\gamma}(s), \mathcal{T}_{d_{\gamma}}(s)\right\}$ as Frenet apparatus of the evolute curve in $S_{1}^{3}$.

Theorem 3 For a given spacelike evolute curve $d_{\gamma}(s)$ of $\gamma(s)$, the Frenet apparatus are introduced as:

$$
\begin{aligned}
\mathbf{T}_{d_{\gamma}}(s)= & \dot{\lambda}_{1} \gamma(s)+\left(\lambda_{1}-\delta \kappa_{g} \lambda_{2}\right) \mathbf{T}(s)+\left(\dot{\lambda}_{2}+\tau_{g} \lambda_{3}\right) \mathbf{N}(s)+\left(\dot{\lambda}_{3}+\tau_{g} \lambda_{2}\right) \mathbf{E}(s), \\
\mathbf{N}_{d_{\gamma}}(s)= & \left(\left|-\left(\xi_{1}+\lambda_{1}\right)^{2}+\xi_{2}^{2}+\left(\xi_{3}+\lambda_{2}\right)^{2}+\left(\xi_{4}+\lambda_{3}\right)^{2}\right|\right)^{\frac{-1}{2}}\left(\left(\left(\xi_{1}+\lambda_{1}\right) \gamma(s)\right.\right. \\
& \left.\left.+\xi_{2} \mathbf{T}(s)+\left(\xi_{3}+\lambda_{2}\right) \mathbf{N}(s)+\left(\xi_{4}+\lambda_{3}\right) \mathbf{E}(s)\right)\right), \\
\mathcal{K}_{d_{\gamma}}= & \sqrt{\left|-\left(\xi_{1}+\lambda_{1}\right)^{2}+\xi_{2}^{2}+\left(\xi_{3}+\lambda_{2}\right)^{2}+\left(\xi_{4}+\lambda_{3}\right)^{2}\right|,} \\
\mathbf{E}_{d_{\gamma}}(s)= & -\frac{1}{\mathcal{K}_{d_{\gamma}}}\left(\left(-\lambda_{2}\left(\left(\lambda_{1}-\kappa_{g} \lambda_{2}\right)\left(\xi_{4}+\lambda_{3}\right)-\xi_{2}\left(\dot{\lambda}_{2}+\tau_{g} \lambda_{3}\right)\right)+\lambda_{3}\left(\left(\lambda_{1}-\kappa_{g} \lambda_{2}\right)\left(\xi_{3}+\lambda_{2}\right)\right.\right.\right. \\
- & \left.\left.\xi_{2}\left(\dot{\lambda}_{2}+\tau_{g} \lambda_{3}\right)\right)\right) \gamma(s)+\left(\lambda_{1}\left(\left(\dot{\lambda}_{2}+\tau_{g} \lambda_{3}\right)\left(\xi_{4}+\lambda_{3}\right)-\left(\xi_{3}+\lambda_{2}\right)\left(\dot{\lambda}_{3}+\tau_{g} \lambda_{2}\right)\right)\right. \\
- & \left.\lambda_{2}\left(\dot{\lambda}_{1}\left(\xi_{4}+\lambda_{3}\right)-\left(\xi_{1}+\lambda_{1}\right)\left(\dot{\lambda}_{3}+\tau_{g} \lambda_{2}\right)\right)+\lambda_{3}\left(\dot{\lambda}_{1}\left(\xi_{3}+\lambda_{2}\right)-\left(\xi_{1}+\lambda_{1}\right)\left(\dot{\lambda}_{2}+\tau_{g} \lambda_{3}\right)\right)\right) \mathbf{T}(s) \\
- & \left(\lambda_{1}\left(\left(\lambda_{1}-\kappa_{g} \lambda_{2}\right)\left(\xi_{4}+\lambda_{3}\right)-\xi_{2}\left(\dot{\lambda}_{3}+\tau_{g} \lambda_{2}\right)\right)+\lambda_{3}\left(\dot{\lambda}_{1} \xi_{2}-\left(\xi_{1}+\lambda_{1}\right)\left(\lambda_{1}-\kappa_{g} \lambda_{2}\right)\right)\right) \mathbf{N}(s) \\
+ & \left.\left(\lambda_{1}\left(\left(\lambda_{1}-\kappa_{g} \lambda_{2}\right)\left(\xi_{3}+\lambda_{2}\right)-\xi_{2}\left(\dot{\lambda}_{2}+\tau_{g} \lambda_{3}\right)\right)+\lambda_{2}\left(\dot{\lambda}_{1} \xi_{2}-\left(\xi_{1}+\lambda_{1}\right)\left(\lambda_{1}-\kappa_{g} \lambda_{2}\right)\right)\right) \mathbf{E}(s)\right),
\end{aligned}
$$




$$
\begin{aligned}
\mathcal{T}_{d_{\gamma}}(s)= & \frac{1}{\mathcal{K}_{d_{\gamma}}^{2}}\left(\lambda_{1}\left(\left(\lambda_{1}-\kappa_{g} \lambda_{2}\right)\left(\xi_{3} B_{4}-\xi_{4} B_{3}\right)-\left(\dot{\lambda}_{2}+\tau_{g} \lambda_{3}\right)\left(\xi_{2} B_{4}-\xi_{4} B_{2}\right)+\left(\dot{\lambda}_{3}+\tau_{g} \lambda_{2}\right)\left(\xi_{2} B_{3}-\xi_{3} B_{2}\right)\right)\right. \\
& -\lambda_{2}\left(\dot{\lambda}_{1}\left(\xi_{2} B_{4}-\xi_{4} B_{2}\right)-\left(\lambda_{1}-\kappa_{g} \lambda_{2}\right)\left(\xi_{1} B_{4}-\xi_{4} B_{1}\right)+\left(\dot{\lambda}_{3}+\tau_{g} \lambda_{2}\right)\left(\xi_{1} B_{2}-\xi_{2} B_{1}\right)\right) \\
& \left.+\lambda_{3}\left(\dot{\lambda}_{1}\left(\xi_{2} B_{3}-\xi_{3} B_{2}\right)-\left(\lambda_{1}-\kappa_{g} \lambda_{2}\right)\left(\xi_{1} B_{3}-\xi_{3} B_{1}\right)+\left(\dot{\lambda}_{2}+\tau_{g} \lambda_{3}\right)\left(\xi_{1} B_{2}-\xi_{2} B_{1}\right)\right)\right),
\end{aligned}
$$

where the functions $\lambda_{1}, \lambda_{2}, \lambda_{3}, \xi_{1}, \xi_{2}, \xi_{3}, \xi_{4}, B_{1}, B_{2}, B_{3}$, and $B_{4}$ being smooth functions.

Proof From the definition of an evolute curve in de Sitter 3-space, we have

$$
d_{\gamma}(s)=\frac{\kappa_{g}}{\sqrt{\left|\left(\frac{\dot{\kappa_{g}}}{\kappa_{g} \tau_{g}}\right)^{2}-\kappa_{g}^{2}+1\right|}} \gamma+\frac{1}{\sqrt{\left|\left(\frac{\dot{\kappa_{g}}}{\kappa_{g} \tau_{g}}\right)^{2}-\kappa_{g}^{2}+1\right|}} \mathbf{N}-\frac{\dot{\kappa_{g}}}{\kappa_{g} \tau_{g} \sqrt{\left|\left(\frac{\dot{\kappa_{g}}}{\kappa_{g} \tau_{g}}\right)^{2}-\kappa_{g}^{2}+1\right|}} \mathbf{E} .
$$

If we denote by

$$
\begin{aligned}
& \lambda_{1}=\frac{\kappa_{g}}{\sqrt{\left|\left(\frac{\dot{\kappa_{g}}}{\kappa_{g} \tau_{g}}\right)^{2}-\kappa_{g}^{2}+1\right|}}, \lambda_{2}=\frac{1}{\sqrt{\left|\left(\frac{\dot{\kappa_{g}}}{\kappa_{g} \tau_{g}}\right)^{2}-\kappa_{g}^{2}+1\right|}}, \\
& \lambda_{3}=-\frac{\dot{\kappa}_{g}}{\kappa_{g} \tau_{g} \sqrt{\left|\left(\frac{\dot{\kappa_{g}}}{\kappa_{g} \tau_{g}}\right)^{2}-\kappa_{g}^{2}+1\right|}}
\end{aligned}
$$

then (22) can be written as

$$
d_{\gamma}(s)=\lambda_{1} \gamma(s)+\lambda_{2} \mathbf{N}(s)+\lambda_{3} \mathbf{E}(s) .
$$

Differentiating both sides of (24), and using (5), we get

$$
\begin{aligned}
\mathbf{T}_{d_{\gamma}}(s)= & \dot{\lambda}_{1} \gamma(s)+\lambda_{1} \dot{\gamma}(s)+\dot{\lambda}_{2} \mathbf{N}(s)+\lambda_{2} \dot{\mathbf{N}}(s)+\dot{\lambda}_{3} \mathbf{E}(s)+\lambda_{3} \dot{\mathbf{E}}(s) \\
= & \dot{\lambda}_{1} \gamma(s)+\lambda_{1} \mathbf{T}(s)+\dot{\lambda}_{2} \mathbf{N}(s)+\lambda_{2}\left(-\delta \kappa_{g} \mathbf{T}(s)+\tau_{g} \mathbf{E}(s)\right) \\
& +\dot{\lambda}_{3} \mathbf{E}(s)+\lambda_{3}\left(\tau_{g} \mathbf{N}(s)\right) \\
= & \dot{\lambda}_{1} \gamma(s)+\left(\lambda_{1}-\delta \kappa_{g} \lambda_{2}\right) \mathbf{T}(s)+\left(\dot{\lambda}_{2}+\tau_{g} \lambda_{3}\right) \mathbf{N}(s) \\
& +\left(\dot{\lambda}_{3}+\tau_{g} \lambda_{2}\right) \mathbf{E}(s),
\end{aligned}
$$

which gives by differentiating

$$
\dot{\mathbf{T}}_{d_{\gamma}}(s)=\left\{\begin{array}{l}
\left(\ddot{\lambda}_{1}-\lambda_{1}+\delta \kappa_{g} \lambda_{2}\right) \gamma(s)+\left(2 \dot{\lambda}_{1}-\delta \dot{\kappa}_{g} \lambda_{2}-2 \delta \kappa_{g} \dot{\lambda}_{2}-\delta \kappa_{g} \tau_{g} \lambda_{3}\right) \mathbf{T}(s) \\
+\left(\kappa_{g} \lambda_{1}-\delta \kappa_{g}^{2} \lambda_{2}+\ddot{\lambda}_{2}+\dot{\tau}_{g} \lambda_{3}+2 \tau_{g} \dot{\lambda}_{3}+\tau_{g}^{2} \lambda_{2}\right) \mathbf{N}(s) \\
+\left(\tau_{g} \dot{\lambda}_{2}+\tau_{g}^{2} \lambda_{3}+\ddot{\lambda}_{3}+\dot{\tau}_{g} \lambda_{2}+\tau_{g} \dot{\lambda}_{2}\right) \mathbf{E}(s) .
\end{array}\right.
$$

In another form, (26) is written

$$
\dot{\mathbf{T}}_{d_{\gamma}}(s)=\xi_{1} \gamma(s)+\xi_{2} \mathbf{T}(s)+\xi_{3} \mathbf{N}(s)+\xi_{4} \mathbf{E}(s),
$$

where

$$
\left\{\begin{array}{l}
\xi_{1}=\left(\ddot{\lambda}_{1}-\lambda_{1}+\delta \kappa_{g} \lambda_{2}\right) \\
\xi_{2}=\left(2 \dot{\lambda}_{1}-\delta \dot{\kappa}_{g} \lambda_{2}-2 \delta \kappa_{g} \dot{\lambda}_{2}-\delta \kappa_{g} \tau_{g} \lambda_{3}\right) \\
\xi_{3}=\left(\kappa_{g} \lambda_{1}-\delta \kappa_{g}^{2} \lambda_{2}+\ddot{\lambda}_{2}+\dot{\tau}_{g} \lambda_{3}+2 \tau_{g} \dot{\lambda}_{3}+\tau_{g}^{2} \lambda_{2}\right) \\
\xi_{4}=\left(\tau_{g} \dot{\lambda}_{2}+\tau_{g}^{2} \lambda_{3}+\ddot{\lambda}_{3}+\dot{\tau}_{g} \lambda_{2}+\tau_{g} \dot{\lambda}_{2}\right) .
\end{array}\right.
$$

Thus, from Eqs. (6), (24), and (27), we obtain 


$$
\begin{aligned}
\mathbf{N}_{d_{\gamma}}(s)= & \left(\left|-\left(\xi_{1}+\lambda_{1}\right)^{2}+\xi_{2}^{2}+\left(\xi_{3}+\lambda_{2}\right)^{2}+\left(\xi_{4}+\lambda_{3}\right)^{2}\right|\right)^{\frac{-1}{2}}\left(\left(\left(\xi_{1}+\lambda_{1}\right) \gamma(s)\right.\right. \\
& \left.\left.+\xi_{2} \mathbf{T}(s)+\left(\xi_{3}+\lambda_{2}\right) \mathbf{N}(s)+\left(\xi_{4}+\lambda_{3}\right) \mathbf{E}(s)\right)\right),
\end{aligned}
$$

and

$$
\mathcal{K}_{d_{\gamma}}=\sqrt{\left|-\left(\xi_{1}+\lambda_{1}\right)^{2}+\xi_{2}^{2}+\left(\xi_{3}+\lambda_{2}\right)^{2}+\left(\xi_{4}+\lambda_{3}\right)^{2}\right|} .
$$

Moreover, by differentiating Eq. (26) with respect to $s$, one can obtain

$$
\begin{aligned}
\dddot{d}_{\gamma}(s)= & \left(\ddot{\lambda}_{1}-3 \dot{\lambda}_{1}+2 \delta \dot{\kappa}_{g} \lambda_{2}+3 \delta \kappa_{g} \dot{\lambda}_{2}+\delta \kappa_{g} \tau_{g} \lambda_{3}\right) \gamma(s)+\left(3 \ddot{\lambda}_{1}-\lambda_{1}+\delta \kappa_{g} \lambda_{2}\right. \\
& -\delta \ddot{\kappa}_{g} \lambda_{2}-3 \delta \dot{\kappa}_{g} \dot{\lambda}_{2}-3 \delta \kappa_{g} \ddot{\lambda}_{2}-\delta \dot{\kappa}_{g} \tau_{g} \lambda_{3}-\delta \kappa_{g} \dot{\tau}_{g} \lambda_{3}-3 \kappa_{g} \tau_{g} \dot{\lambda}_{3}-\delta \kappa_{g}^{2} \lambda_{1} \\
& \left.+\delta \kappa_{g}^{3} \lambda_{2}-\delta \kappa_{g} \tau_{g}^{2} \lambda_{2}\right) \mathbf{T}(s)+\left(3 \kappa_{g} \dot{\lambda}_{1}-3 \delta \kappa_{g} \dot{\kappa}_{g} \lambda_{2}-3 \delta \kappa_{g}^{2} \dot{\lambda}_{2}+\dot{\kappa}_{g} \lambda_{1}\right. \\
& -\delta \kappa_{g}^{2} \tau_{g} \lambda_{3}+3 \tau_{g} \dot{\tau}_{g} \lambda_{2}+3 \tau_{g}^{2} \dot{\lambda}_{2}+\dddot{\lambda}_{2}+\ddot{\tau}_{g} \lambda_{3}+3 \dot{\tau}_{g} \dot{\lambda}_{3}+\tau_{g} \ddot{\lambda}_{3}+\tau_{g}^{3} \lambda_{3} \\
& \left.+2 \dot{\tau}_{g} \ddot{\lambda}_{3}\right) \mathbf{N}(s)+\left(\kappa_{g} \tau_{g} \lambda_{1}-\delta \kappa_{g}^{2} \tau_{g} \lambda_{2}+\tau_{g}^{3} \lambda_{2}+3 \tau_{g} \ddot{\lambda}_{2}+3 \tau_{g} \dot{\tau}_{g} \lambda_{3}+\dddot{\lambda}_{3}\right. \\
& \left.+3 \tau_{g}^{2} \dot{\lambda}_{3}+3 \dot{\tau}_{g} \dot{\lambda}_{2}+\ddot{\tau}_{g} \lambda_{2}\right) \mathbf{E}(s),
\end{aligned}
$$

which in abbreviated form

$$
\dddot{d}_{\gamma}(s)=B_{1} \gamma(s)+B_{2} \mathbf{T}(s)+B_{3} \mathbf{N}(s)+B_{4} \mathbf{E}(s),
$$

where

$$
\left\{\begin{aligned}
B_{1} & =\left(\ddot{\lambda}_{1}-3 \dot{\lambda}_{1}+2 \delta \dot{\kappa}_{g} \lambda_{2}+3 \delta \kappa_{g} \dot{\lambda}_{2}+\delta \kappa_{g} \tau_{g} \lambda_{3}\right) \\
B_{2} & =\left(3 \ddot{\lambda}_{1}-\lambda_{1}+\delta \kappa_{g} \lambda_{2}-\delta \ddot{\kappa}_{g} \lambda_{2}-3 \delta \dot{\kappa}_{g} \dot{\lambda}_{2}-3 \delta \kappa_{g} \ddot{\lambda}_{2}-\delta \dot{\kappa}_{g} \tau_{g} \lambda_{3}\right. \\
& \left.-\delta \kappa_{g} \dot{\tau}_{g} \lambda_{3}-3 \kappa_{g} \tau_{g} \dot{\lambda}_{3}-\delta \kappa_{g}^{2} \lambda_{1}+\delta \kappa_{g}^{3} \lambda_{2}-\delta \kappa_{g} \tau_{g}^{2} \lambda_{2}\right) \\
B_{3} & =\left(3 \kappa_{g} \dot{\lambda}_{1}-3 \delta \kappa_{g} \dot{\kappa}_{g} \lambda_{2}-3 \delta \kappa_{g}^{2} \dot{\lambda}_{2}+\dot{\kappa}_{g} \lambda_{1}-\delta \kappa_{g}^{2} \tau_{g} \lambda_{3}+3 \tau_{g} \dot{\tau}_{g} \lambda_{2}\right. \\
& \left.+3 \tau_{g}^{2} \dot{\lambda}_{2}+\ddot{\lambda}_{2}+\ddot{\tau}_{g} \lambda_{3}+3 \dot{\tau}_{g} \dot{\lambda}_{3}+\tau_{g} \ddot{\lambda}_{3}+\tau_{g}^{3} \lambda_{3}+2 \dot{\tau}_{g} \ddot{\lambda}_{3}\right) \\
B_{4} & =\left(\kappa_{g} \tau_{g} \lambda_{1}-\delta \kappa_{g}^{2} \tau_{g} \lambda_{2}+\tau_{g}^{3} \lambda_{2}+3 \tau_{g} \ddot{\lambda}_{2}+3 \tau_{g} \dot{\tau}_{g} \lambda_{3}+\dddot{\lambda}_{3}+3 \tau_{g}^{2} \dot{\lambda}_{3}\right. \\
& \left.+3 \dot{\tau}_{g} \dot{\lambda}_{2}+\ddot{\tau}_{g} \lambda_{2}\right) .
\end{aligned}\right.
$$

Now, after using (24), (25), (27), and (30), the torsion $\mathcal{T}_{d_{\gamma}}$ of the evolute curve $d_{\gamma}(s)$ is obtained

$$
\begin{aligned}
\mathcal{T}_{d_{\gamma}}(s)= & \frac{1}{\mathcal{K}_{d_{\gamma}}^{2}}\left(\lambda_{1}\left(\left(\lambda_{1}-\kappa_{g} \lambda_{2}\right)\left(\xi_{3} B_{4}-\xi_{4} B_{3}\right)-\left(\dot{\lambda}_{2}+\tau_{g} \lambda_{3}\right)\left(\xi_{2} B_{4}-\xi_{4} B_{2}\right)+\left(\dot{\lambda}_{3}+\tau_{g} \lambda_{2}\right)\left(\xi_{2} B_{3}-\xi_{3} B_{2}\right)\right)\right. \\
& -\lambda_{2}\left(\dot{\lambda}_{1}\left(\xi_{2} B_{4}-\xi_{4} B_{2}\right)-\left(\lambda_{1}-\kappa_{g} \lambda_{2}\right)\left(\xi_{1} B_{4}-\xi_{4} B_{1}\right)+\left(\dot{\lambda}_{3}+\tau_{g} \lambda_{2}\right)\left(\xi_{1} B_{2}-\xi_{2} B_{1}\right)\right) \\
& \left.+\lambda_{3}\left(\dot{\lambda}_{1}\left(\xi_{2} B_{3}-\xi_{3} B_{2}\right)-\left(\lambda_{1}-\kappa_{g} \lambda_{2}\right)\left(\xi_{1} B_{3}-\xi_{3} B_{1}\right)+\left(\dot{\lambda}_{2}+\tau_{g} \lambda_{3}\right)\left(\xi_{1} B_{2}-\xi_{2} B_{1}\right)\right)\right) .
\end{aligned}
$$

The only vector remaining from Frenet apparatus is the binormal vector $\mathbf{E}_{\gamma}(s)$, which is given directly by

$$
\begin{aligned}
\mathbf{E}_{d_{\gamma}}(s)=-\frac{1}{\mathcal{K}_{d_{\gamma}}}\left|\begin{array}{ccc}
0 & \lambda_{2} & \lambda_{3} \\
\left(\lambda_{1}-\kappa_{g} \lambda_{2}\right) & \left(\dot{\lambda}_{2}+\tau_{g} \lambda_{3}\right) & \left(\dot{\lambda}_{3}+\tau_{g} \lambda_{2}\right) \\
\xi_{2} & \left(\xi_{3}+\lambda_{2}\right) & \left(\xi_{4}+\lambda_{3}\right)
\end{array}\right| \gamma(s) \\
-\frac{1}{\mathcal{K}_{d_{\gamma}}}\left|\begin{array}{ccc}
\lambda_{1} & \lambda_{2} & \lambda_{3} \\
\dot{\lambda}_{1} & \left(\dot{\lambda}_{2}+\tau_{g} \lambda_{3}\right) & \left(\dot{\lambda}_{3}+\tau_{g} \lambda_{2}\right) \\
\left(\xi_{1}+\lambda_{1}\right) & \left(\xi_{3}+\lambda_{2}\right) & \left(\xi_{4}+\lambda_{3}\right)
\end{array}\right| \mathbf{T}(s)
\end{aligned}
$$




$$
\begin{gathered}
+\frac{1}{\mathcal{K}_{d_{\gamma}}}\left|\begin{array}{ccc}
\lambda_{1} & 0 & \lambda_{3} \\
\dot{\lambda}_{1} & \left(\lambda_{1}-\kappa_{g} \lambda_{2}\right) & \left(\dot{\lambda}_{3}+\tau_{g} \lambda_{2}\right) \\
\left(\xi_{1}+\lambda_{1}\right) & \xi_{2} & \left(\xi_{4}+\lambda_{3}\right)
\end{array}\right| \mathbf{N}(s) \\
-\frac{1}{\mathcal{K}_{d_{\gamma}}}\left|\begin{array}{ccc}
\lambda_{1} & 0 & \lambda_{2} \\
\dot{\lambda}_{1} & \left(\lambda_{1}-\kappa_{g} \lambda_{2}\right) & \left(\dot{\lambda}_{2}+\tau_{g} \lambda_{3}\right) \\
\left(\xi_{1}+\lambda_{1}\right) & \xi_{2} & \left(\xi_{3}+\lambda_{2}\right)
\end{array}\right| \mathbf{E}(s),
\end{gathered}
$$

Thus, the theorem has been proven.

\section{Examples}

In this section, to support the theoretical results of this paper, we construct two examples of an evolute curve in the two- and three-dimensional hyperbolic spaces, then we calculate its Frenet apparatus using Frenet apparatus of its involute curve.

Example 1 Consider the general helix curve $\alpha$ in $H_{+}^{2}(-1)$, where

$$
\alpha(s)=\left(\frac{s}{\sqrt{2}}, \sin \left(\sqrt{\frac{3}{2}} s\right), \cos \left(\sqrt{\frac{3}{2}} s\right)\right) .
$$

Firstly, we compute the Frenet frame, the curvature and the torsion of the curve $\alpha$. For this, from Eq. (33), the tangent vector of the curve $\alpha$ is given by

$$
\mathbf{T}(s)=\dot{\alpha}(s)=\left(\frac{1}{\sqrt{2}}, \sqrt{\frac{3}{2}} \cos \left(\sqrt{\frac{3}{2}} s\right),-\sqrt{\frac{3}{2}} \sin \left(\sqrt{\frac{3}{2}} s\right)\right) .
$$

And from (33) and (34), we get

$$
\alpha(s) \wedge \mathbf{T}(s)=\left|\begin{array}{ccc}
-i & j & k \\
\frac{s}{\sqrt{2}} & \sin \left(\sqrt{\frac{3}{2}} s\right) & \cos \left(\sqrt{\frac{3}{2}} s\right) \\
\frac{1}{\sqrt{2}} & \sqrt{\frac{3}{2}} \cos \left(\sqrt{\frac{3}{2}} s\right) & -\sqrt{\frac{3}{2}} \sin \left(\sqrt{\frac{3}{2}} s\right)
\end{array}\right|,
$$

which enable us to obtain

$$
\begin{aligned}
\mathbf{E}(s)= & \left(\sqrt{\frac{3}{2}}\right) i+\left(\frac{\sqrt{3}}{2} s \sin \left(\sqrt{\frac{3}{2}} s\right)+\frac{1}{\sqrt{2}} \cos \left(\sqrt{\frac{3}{2}} s\right)\right) j \\
& +\left(\frac{\sqrt{3}}{2} s \cos \left(\sqrt{\frac{3}{2}} s\right)-\frac{1}{\sqrt{2}} \sin \left(\sqrt{\frac{3}{2}} s\right)\right) k
\end{aligned}
$$

the curvature of the curve $\alpha(s)$ is given as follows:

$$
\begin{aligned}
\kappa_{g}(s)= & -\frac{s}{\sqrt{2}}\left(\frac{3}{2} \sqrt{\frac{3}{2}} \cos ^{2}\left(\sqrt{\frac{3}{2}} s\right)+\frac{3}{2} \sqrt{\frac{3}{2}} \sin ^{2}\left(\sqrt{\frac{3}{2}} s\right)\right) \\
& +\frac{3}{2 \sqrt{2}}\left(\sin \left(\sqrt{\frac{3}{2}} s\right) \cos \left(\sqrt{\frac{3}{2}} s\right)-\sin \left(\sqrt{\frac{3}{2}} s\right) \cos \left(\sqrt{\frac{3}{2}} s\right)\right),
\end{aligned}
$$

which takes the simple form

$$
\kappa_{g}(s)=-\frac{\sqrt{27}}{4} s .
$$


Thus, from Theorem (1), the Frenet apparatus of the evolute curve $h_{\alpha}$ of $\alpha(s)$ (see Figs. 1 and 2) are expressed respectively, by

$$
\begin{aligned}
& h_{\alpha}(s)=\frac{1}{\sqrt{\left|27 s^{2}-16\right|}}\left\{\begin{array}{l}
\left(\sqrt{\frac{3}{2}}\left(4-3 s^{2}\right), \frac{4}{\sqrt{2}} \cos \left(\sqrt{\frac{3}{2}} s\right)-\sqrt{3} s \sin \left(\sqrt{\frac{3}{2}} s\right)\right. \\
\left.,-\frac{4}{\sqrt{2}} \sin \left(\sqrt{\frac{3}{2}} s\right)-\sqrt{3} s \cos \left(\sqrt{\frac{3}{2}} s\right)\right),
\end{array}\right. \\
& \mathbf{T}_{h_{\alpha}}(s)=\frac{6 \sqrt{3}}{\left|27 s^{2}-16\right|^{\frac{3}{2}}}\left\{\begin{array}{l}
\left(-5 \sqrt{2} s,\left(9 s^{2}-2\right) \sin \left(\sqrt{\frac{3}{2}} s\right)-3 \sqrt{6} s \cos \left(\sqrt{\frac{3}{2}} s\right)\right. \\
\left.,\left(9 s^{2}-2\right) \cos \left(\sqrt{\frac{3}{2}} s\right)+3 \sqrt{6} s \sin \left(\sqrt{\frac{3}{2}} s\right)\right),
\end{array}\right. \\
& \mathbf{E}_{h_{\alpha}}(s)=\frac{-12 \sqrt{3}}{\left|27 s^{2}-16\right|}\left(\frac{1}{\sqrt{2}}, \sqrt{\frac{3}{2}} \cos \left(\sqrt{\frac{3}{2}} s\right),-\sqrt{\frac{3}{2}} \sin \left(\sqrt{\frac{3}{2}} s\right)\right),
\end{aligned}
$$

and

$$
\mathcal{K}_{h_{\alpha}}(s)=\frac{27}{\left|27 s^{2}-16\right|^{\frac{3}{2}}} .
$$

Example 2 Let $\beta$ be a general helix in $H_{+}^{3}(-1)$, where

$$
\beta(s)=(\sqrt{2} \cosh (s), \sqrt{2} \sinh (s), \sin (s), \cos (s)) .
$$

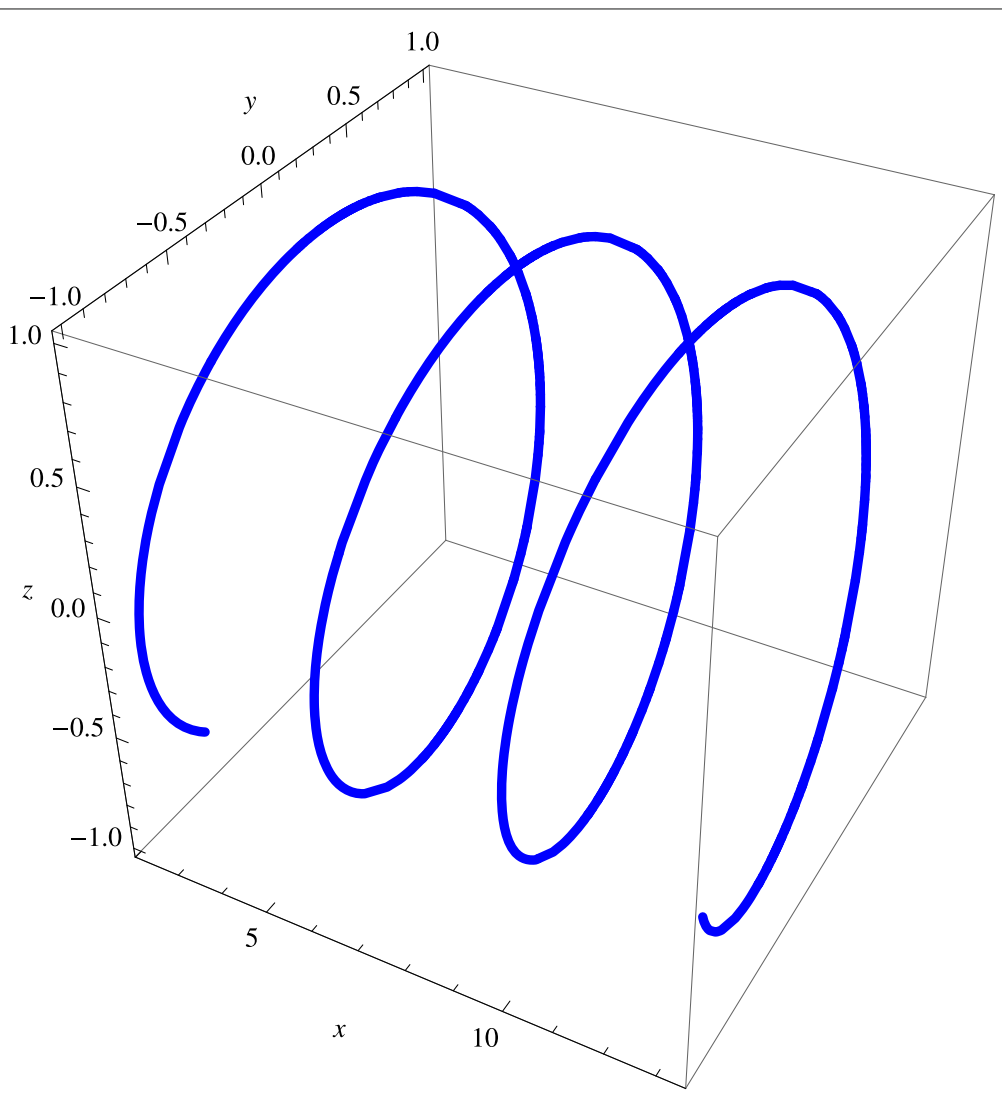

Fig. 1 Evolute curve $h_{\alpha}(s)$ 


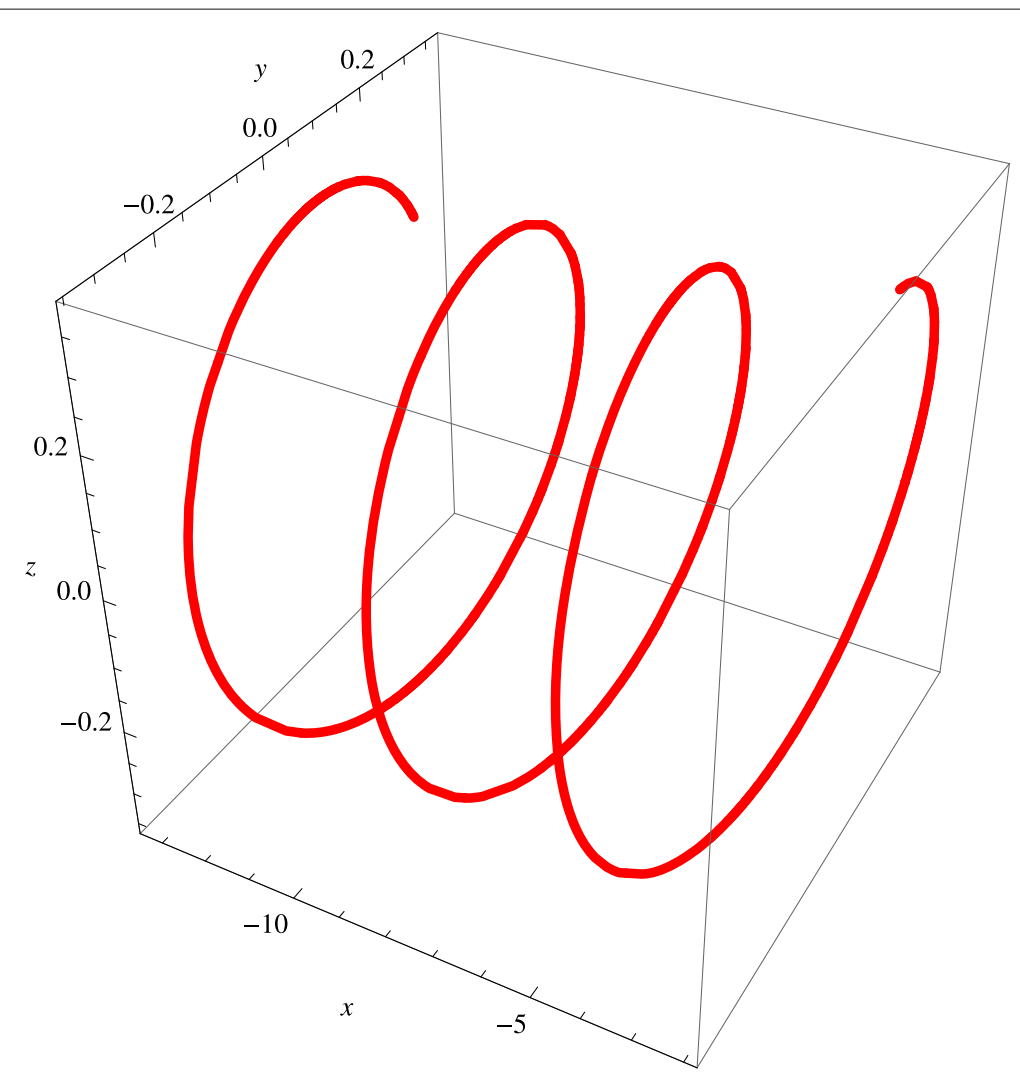

Fig. 2 Involute curve $\alpha(s)$

From (37), the tangent vector of the curve $\beta$ is given by

$$
\mathbf{T}(s)=(\sqrt{2} \sinh (s), \sqrt{2} \cosh (s), \cos (s),-\sin (s)),
$$

which gives by differentiating

$$
\dot{\mathbf{T}}(s)=(\sqrt{2} \cosh (s), \sqrt{2} \sinh (s),-\sin (s),-\cos (s)) .
$$

From Eqs. (37) and (39), we get

$$
\mathbf{N}(s)=\frac{\dot{\mathbf{T}}(s)-\beta(s)}{\|\dot{\mathbf{T}}(s)-\beta(s)\|}=(0,0,-\sin (s),-\cos (s)),
$$

and

$$
\kappa_{g}(s)=\|\dot{\mathbf{T}}(s)-\beta(s)\|=2 .
$$

Also, we get

$$
\mathbf{E}(s)=(\sqrt{2} \sinh (s), \sqrt{2} \cosh (s),-2 \cos (s), 2 \sin (s)) .
$$


Using the differentiation of Eq. (37) three times with respect to s to obtain

$$
\begin{aligned}
\operatorname{det}(\beta ; \dot{\beta} ; \ddot{\beta} ; \dddot{\beta})= & \sqrt{2} \cosh (s)[-\sqrt{2} \cosh (s)-\cos (s)(\sqrt{2} \sin (s) \sinh (s) \\
& +\sqrt{2} \cos (s) \cosh (s))-\sin (s)(-\sqrt{2} \cos (s) \sinh (s) \\
& +\sqrt{2} \sin (s) \cosh (s))]-\sqrt{2} \sinh (s)[-\sqrt{2} \sinh (s) \\
& -\cos (s)(\sqrt{2} \sin (s) \cosh (s)+\sqrt{2} \cos (s) \sinh (s)) \\
& -\sin (s)(-\sqrt{2} \cos (s) \cosh (s)+\sqrt{2} \sin (s) \sinh (s)] \\
& +\sin (s)[\sqrt{2} \sinh (s)(\sqrt{2} \sin (s) \sinh (s)+\sqrt{2} \cos (s) \cosh (s)) \\
& -\sqrt{2} \cosh (s)(\sqrt{2} \sin (s) \cosh (s)+\sqrt{2} \cos (s) \sinh (s)) \\
& -2 \sin (s))]-\cos (s)[\sqrt{2} \sinh (s)(-\sqrt{2} \cos (s) \sinh (s) \\
& +\sqrt{2} \sin (s) \cosh (s))-\sqrt{2} \cosh (s)(-\sqrt{2} \cos (s) \cosh (s) \\
& +\sqrt{2} \sin (s) \sinh (s))+2 \cos (s))]
\end{aligned}
$$

then, we get

$$
\tau_{g}(s)=-\frac{\operatorname{det}(\beta, \dot{\beta}, \ddot{\beta}, \dddot{\beta})}{\kappa_{g}^{2}}=2 .
$$

From Theorem (2) and Eqs. (11), (16) and (20), the Frenet apparatus of the evolute curve $h_{\beta}$ of $\beta(s)$ are respectively, expressed by

$$
\begin{aligned}
& h_{\beta}(s)=\frac{1}{\sqrt{3}}(2 \sqrt{2} \cosh (s), 2 \sqrt{2} \sinh (s), \sin (s), \cos (s)), \\
& \left\{\begin{array}{l}
\mu_{1}=\frac{2}{\sqrt{3}}, \mu_{2}=\frac{1}{\sqrt{3}}, \mu_{3}=0 \\
\eta_{1}=\eta_{2}=\eta_{4}=0, \quad \eta_{3}=\frac{-4}{3} \\
\zeta_{1}=\zeta_{2}=\zeta_{3}=0, \quad \zeta_{4}=\frac{-8}{\sqrt{3}}
\end{array}\right. \\
& \mathbf{T}_{h_{\beta}}(s)=\frac{2}{\sqrt{3}}(\sqrt{2} \sinh (s), \sqrt{2} \cosh (s),-2 \cos (s), 2 \sin (s)), \\
& \mathbf{N}_{h_{\beta}}(s)=\frac{-1}{\sqrt{5}}(-2 \sinh (s),-2 \cosh (s),(-2 \sqrt{2}+5) \sin (s),(-2 \sqrt{2}+5) \cos (s)), \\
& \mathbf{E}_{h_{\beta}}(s)=\frac{-16}{3 \sqrt{5}}(\sqrt{2} \sinh (s), \sqrt{2} \cosh (s), \cos (s),-\sin (s)),
\end{aligned}
$$

and

$$
\mathcal{K}_{h_{\beta}}(s)=\sqrt{\frac{5}{3}}, \quad \mathcal{T}_{h_{\beta}}(s)=0 .
$$

\section{Acknowledgements}

The authors are very grateful to referees for the useful suggestions and remarks for the revised version.

\section{Authors' contributions}

All authors read and approved the final manuscript.

\section{Funding}

Not applicable.

\section{Availability of data and materials}

Not applicable. 
Received: 22 March 2019 Accepted: 26 June 2019

Published online: 02 August 2019

\section{References}

1. Bilici, M., Çaliş, M.: Some new notes on the involutes of the timelike curves in Minkowski 3-Space. Int. J. Contemp. Math. Sci. 6, 2019-2030 (2011)

2. Grabiner, J. V.: A History of Mathematics. Wiley, New York (1968)

3. Özyilmaz, E., Yilmaz, S.: Involute-Evolute curve couples in the Euclidean 4-space. Int. J. Open Probl. Compt. Math. 2(2), 168-174 (2009)

4. Sato, T.: Pseudo-spherical evolutes of curves on a spacelike surface in three dimensional Lorentz-Minkowski space. J. Geom. 103, 319-331 (2012)

5. Turgut, M., Yilmaz, S.: On the Frenet frame and a characterization of space-like involute-evolute curve couple in Minkowski space-time. Int. Math. Forum. 16, 793-801 (2008)

6. Abdel-Aziz, H. S., Saad, M. K., Abdel-salam, A. A.: Some geometric invariants of pseudo-spherical evolutes in the hyperbolic 3-space. Comput. Mater. Contin. 57(3), 389-415 (2018)

7. Hayashi, R., Izumiya, S., Sato, T.: Duals of curves in hyperbolic space. Note Mat. 33, 97-106 (2013)

8. Boyer, C.: A History of Mathematics. Wiley, New York (1968)

9. Hayashi, R., Izumiya, S., Sato, T.: Focal surfaces and evolutes of curves in hyperbolic space. Commun. Korean Math. Soc. 32, 147-163 (2017)

10. Izumiya, S., He pei, D., Sano, T., Torii, E.: Evolutes of hyperbolic plane curves. Acta Math. Sin. 20(3), 543-550 (2004)

11. Izumiya, S., Nabarro, C., de jesus, A.: Horospherical and hyperbolic dual surfaces of space-like curves in de sitter space. J. Singularities. 16, 180-193 (2017)

12. Liu, H.: Curves in three dimensional Riemannian space forms. Results Math. 66(3), 469-480 (2014)

\section{Publisher's Note}

Springer Nature remains neutral with regard to jurisdictional claims in published maps and institutional affiliations.

\section{Submit your manuscript to a SpringerOpen ${ }^{\circ}$ journal and benefit from:}

- Convenient online submission

- Rigorous peer review

- Open access: articles freely available online

- High visibility within the field

- Retaining the copyright to your article

Submit your next manuscript at $\gg$ springeropen.com 